Review

\title{
Exploring inhalable polymeric dry powders for anti-tuberculosis drug delivery
}

\author{
Margarida S. Miranda ${ }^{\mathrm{a}, \mathrm{b}, \mathrm{d}}$, Márcia T. Rodrigues ${ }^{\mathrm{a}, \mathrm{b}, \mathrm{d}}$, Rui M.A. Domingues ${ }^{\mathrm{a}, \mathrm{b}, \mathrm{d}}$, Egídio Torrado ${ }^{\mathrm{b}, \mathrm{c}}$, \\ Rui L. Reis ${ }^{\mathrm{a}, \mathrm{b}, \mathrm{d}}$, Jorge Pedrosa ${ }^{\mathrm{b}, \mathrm{c}}$, Manuela E. Gomes ${ }^{\mathrm{a}, \mathrm{b}, \mathrm{d}, *}$ \\ a 3B's Research Group, I3Bs - Research Institute on Biomaterials, Biodegradables and Biomimetics, University of Minho, Headquarters of the European Institute of \\ Excellence on Tissue Engineering and Regenerative Medicine, Avepark - Parque de Ciência e Tecnologia, Zona Industrial da Gandra, 4805-017 Barco, Guimarães, Portugal \\ b ICVS/3B's - PT Government Associate Laboratory, Braga/Guimarães, Portugal \\ ${ }^{\mathrm{c}}$ Life and Health Sciences Research Institute (ICVS), School of Medicine, University of Minho, Braga, Portugal \\ d The Discoveries Centre for Regenerative and Precision Medicine, Headquarters at University of Minho, Avepark, 4805-017 Barco, Guimarães, Portugal
}

\section{A R T I C L E I N F O}

\section{Keywords:}

Polymeric materials

Dry powders

Microparticulate systems

Drug targeting

Alveolar macrophages

Tuberculosis

\begin{abstract}
A B S T R A C T
The growing interest on polymeric delivery systems for pulmonary administration of drugs anticipates a more direct and efficient treatment of diseases such as tuberculosis (TB) that uses the pulmonary route as the natural route of infection. Polymeric microparticles or nano-in-microparticles offer target delivery of drugs to the lungs and the potential to control and sustain drug release within TB infected macrophages improving the efficiency of the anti-TB treatment and reducing side effects. In a dry powder form these inhalable delivery systems have increased stability and prolonged storage time without requiring refrigeration, besides being cost-effective and patient convenient.

Thus, this review aims to compile the recent innovations of inhalable polymeric dry powder systems for the delivery of anti-TB drugs exploring the methods of production, aerodynamic characterization and the efficacy of targeted drug delivery systems using in vitro and in vivo models of the disease. Advanced knowledge and promising outcomes of these systems are anticipated to simplify and revolutionize the pulmonary drug delivery and to contribute towards more effective anti-TB treatments.
\end{abstract}

\section{Introduction}

Tuberculosis (TB), caused by infections with Mycobacterium tuberculosis (Mtb), is an infectious disease of enormous public health impact. TB is an airborne disease initiated by the inhalation of infected droplets that travel the upper respiratory tract and bronchi, being deposited in the lower airways. Here, Mtb is thought to be recognized and phagocytosed by macrophages, specifically alveolar macrophages. In the majority of cases, this initial interaction between Mtb and immune cells results in the development of a host protective response capable of eliminating the invading pathogen. In some cases however, the immune response does not eliminate the infection thereby resulting

\footnotetext{
Abbreviations ${ }^{1}$ : ALG, alginate; Cm, capreomycin; CHI, chitosan; DPIs, dry powder inhalers; E, ethambutol; EC, ethyl cellulose; ECM, extracellular matrix; Eto,

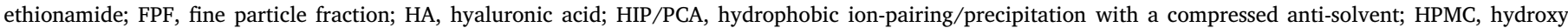

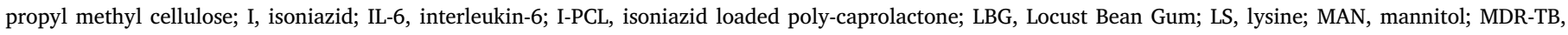

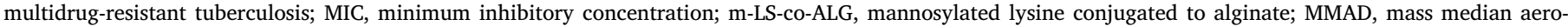

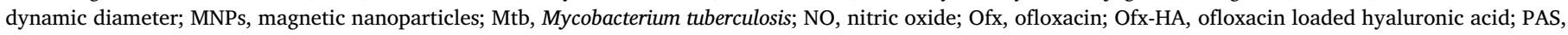

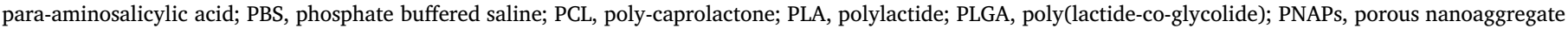

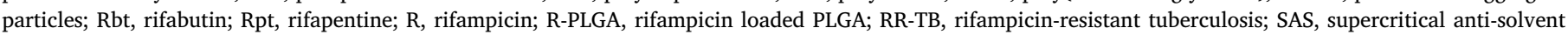

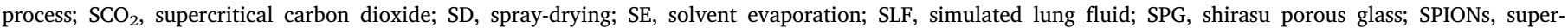

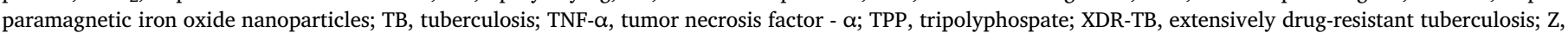
pyrazinamide

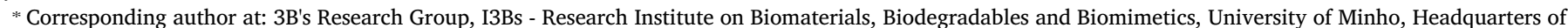

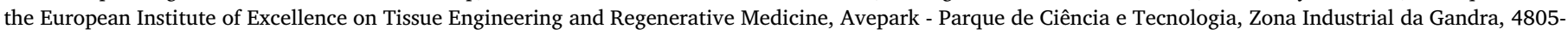
017 Barco, Guimarães, Portugal.

E-mail address: megomes@i3bs.uminho.pt (M.E. Gomes).

${ }^{1}$ Drug abbreviation is in accordance to WHO nomenclature.
} 


\section{Mechanism of deposition of particulate systems}

\section{Impaction} Deposition on upper airways;
digested or exhaled

\section{Sedimentation}

Deposition on lower airways; range within macrophage phagocytosis

\section{(Brownian) Diffusion}

High probability of exhalation; requires a carrier to deposit and uptake in alveoli

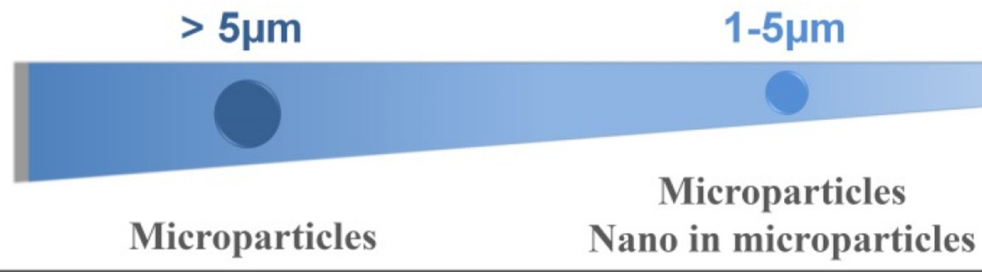

Microparticles

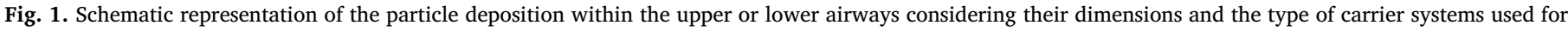
their delivery.

in a spectrum of disease with different phenotypes and clinical manifestations [1]. It is estimated that approximately one-third of the world population has contacted with Mtb and part of this population is likely latently infected [2]. Although these individuals do not show signs or symptoms of TB, as the bacteria proliferation is under immune control, they have a $10 \%$ increased risk of progressing into active TB during their life time being therefore important reservoirs for transmission [3].

The current anti-TB therapy protocols comprise of a six-month combination course of rifampicin (R), isoniazid (I), pyrazinamide (Z) and ethambutol (E), which are first-line anti-TB drugs. All four drugs are taken during the first two months of the treatment following a period of four months where only rifampicin and isoniazid are taken. Although the success rate of this regimen has been estimated to be over $85 \%$ [2], the full length of treatment is crucial for the effective and complete eradication of the pathogen. However, the incorrect use of anti-TB drugs or the use of ineffective drug formulations such as single drug regimen, poor quality medicines or inappropriate storage conditions and premature treatment interruption due to the long duration of the therapeutic regimen and the associated toxic side effects, lead to drug resistance [2].

Treatment for drug resistant strains, which include rifampicin-resistant TB (RR-TB), multidrug-resistant TB (MDR-TB) and extensively drug-resistant TB (XDR-TB) is longer (up to 2 years) and requires more expensive and more toxic drugs with treatment success rates lower than $54 \%$ [2].

Therefore, in addition to classical drug discovery strategies, new approaches are urgently needed to get a faster, more efficient and less harmful treatment. In this regard, the development of novel therapies aiming at pulmonary delivery and drug targeting to the site of infection could be a promising solution to allow a sustainable and controlled release of medicines with therapeutic action, while decreasing the dosage and frequency of conventional chemotherapy and minimizing side effects. Moreover, these approaches may lead to a higher efficiency of the treatment and to a higher patient compliance, minimizing the risk of therapy failure and the development of drug resistant strains.

\section{Delivery of anti-tuberculosis drugs to the lungs}

\subsection{Advantages of the pulmonary route}

Despite the natural barriers to prevent invasion of unwanted airborne particles or living entities, such as airway geometry, humidity, mucociliary clearance and resident populations of macrophages, the lungs are constantly challenged with infectious agents, including Mtb, which make these air-filled organs an attractive target for drug delivery strategies as it provides direct access to the infection site. Delivery systems for TB treatment through the pulmonary route present advantages when compared to the conventional oral and injectable routes. These include drug delivery directly to the infected area, increasing local drug concentration which will impact in bacterial burden and reduce the systemic dosage of the drug [4-13]. Additionally, inhalable systems avoid unwanted side effects often caused by drug metabolism in the gastrointestinal tract before the drug reaches the systemic circulation [4-13]. Unlike the injectable route, inhalation is a pain free and self-administrable delivery means favoring patient convenience and compliance to the treatment.

\subsection{Particulate carriers as inhalable delivery systems}

Pure drug formulations are typically burst released in the lungs and rapidly undergo unspecific distribution [5,14-18]. In order to achieve a more efficient solution, therapeutic agents can be formulated into particulate carrier systems such as microparticles, nanoparticles, liposomes, micelles or dendrimers. Such systems allow protection of drugs from direct contact with the lung tissue, avoiding early degradation, preclude rapid clearance from the body and assist the control and sustain release of drugs over long periods of time. Particulate carriers also reduce drug toxicity, circumvent undesirable physicochemical properties of the drugs (e.g. low water solubility) and improve drug uptake by macrophages [19].

In recent years the advantages of inhalable particulate carrier systems have been allied to the benefits of dry powder formulations to be delivered by dry powder inhalers (DPIs) for an improved drug delivery system. Dry powder formulations have improved stability as a result of its dry form, do not require refrigeration and allow longer storage periods. Formulations are often combined with pharmaceutically suitable excipients such as lactose, leucine, mannitol or trehalose to improve processing and aerodynamic properties [20,21]. DPIs are propellant free, portable, easy to use and cost-effective devices. Moreover, DPIs are activated by the inspiration effort of patients, allowing a rapid and higher dose administration and a more efficient pulmonary drug deposition [22,23]. Thus, DPIs are currently considered the most convenient and suitable alternative for inhaling anti-TB drugs [24].

\subsection{Design and features of inhalable particulate systems}

Medicinal particles are designed and developed so their deposition in the respiratory tract can be predicted rather precisely (Fig. 1). The 


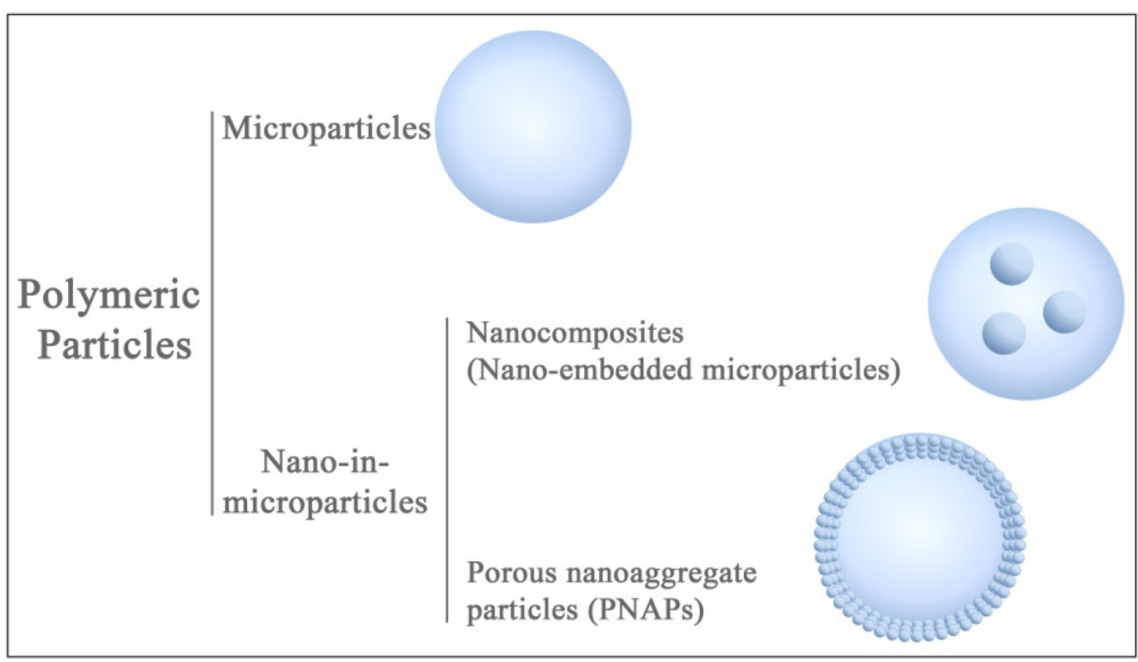

Fig. 2. Developed inhalable dry powder systems for anti-TB drug delivery based on polymeric particles.

behavior of inhaled particles and their deposition depend of several parameters including particle dimensions, density, shape, composition, concentration, surface properties such as particle charge and the breathing pattern (air flow) of the individual $[13,25]$.

The aerodynamic diameter is an important parameter affecting the pulmonary trajectory and depends on both particle dimension and density. Particles with an aerodynamic diameter in the range 1-5 $\mu \mathrm{m}$ are deposited in the lower airways, which is a desirable location for anti-TB drugs [13,26-28]. On the other hand, particles larger than $5 \mu \mathrm{m}$ undergo deposition in the upper regions, such as the nasopharynx and they are consequently swallowed, while particles smaller than $1 \mu \mathrm{m}$ are likely to be exhaled or retained in the alveoli if transported by a carrier system [13,26,29]. After reaching the lower airways, particulate systems are likely to be either phagocytized by alveolar macrophages, adhere to the lung tissue or enter the blood circulation via lung vascular tree. Another potential destination is the entrapment of these particles within the granulomas which are compact and organized aggregates of immune cells where Mtb persists [30,31].

\subsection{Types of microparticulate dry powder systems}

Liposomes, micelles and polymeric particles have been developed as inhalable dry powder particulate systems aiming at anti-TB drug delivery strategies. Liposomes and micelles studies were reviewed elsewhere $[32,33]$. Polymeric particles offer great opportunities in target drug delivery and controlled and sustained release of drugs. The possibility of surface modification of polymeric particles can give rise to systems with a great diversity of physicochemical properties and, if desired, favor a more efficient targeting approach [34]. Additionally, the production of polymeric particles is considerably more cost-effective than lipidic systems.

Polymeric particles include microparticles and nano-in-microparticles and these can further be divided into nanocomposites (also referred as nano-embedded microparticles) and porous nanoaggregate particles (PNAPs) (Fig. 2). Nano-in-microparticle systems have been developed to utilize nanoparticulate systems, often exhaled from the lungs, into successful inhalable drug carrier systems (Fig. 1). In nanocomposites, drug loaded nanoparticles are incorporated in a micronized sugar matrix (e.g. lactose, mannitol, leucine, maltodextrin) to form micro-scaled particles of $1-5 \mu \mathrm{m}$. These nanocomposites can then be decomposed into nanoparticles in the lower airways, since the sugar moiety is soluble in the lung lining fluid. On the other hand, PNAPs consist of spherical nanoaggregates at the micron-size level that also redisperse into the elementary nanoparticles in the lung lining fluid.

A considerable focus has been given to synthetic polymers poly (lactide-co-glycolide) (PLGA) and polylactide (PLA) due to their biocompatible and biodegradable properties, non-toxicity [35-37] and to the ability to encapsulate hydrophobic anti-TB drugs. Synthetic polymers also benefit from high purity products and batch-to-batch uniformity. However, more recently, the trend for dry powder production has been shifted towards the use of natural polymers such as chitosan, gelatin or guar gum due to their abundance and availability in nature, cost effectiveness and the fact that these raw materials require a minimal use of organic solvents to be processed. Also, combinations of polymers have been tested to merge properties of the polymers involved in order to obtain more efficient delivery systems.

\subsection{Techniques used to develop polymeric microparticulate dry powders}

A brief explanation on the most common production techniques to prepare inhalable polymeric drug delivery microparticulate and nanoin-microparticulate systems is described in Table 1 . The techniques presented in this table have shown to produce carrier systems with suitable aerodynamic diameters using a wide range of synthetic and natural based polymers.

Emulsion followed by solvent evaporation is commonly used to produce microparticles, in particular PLGA microparticles, however these are produced with a relatively wide size distribution [38]. Makino et al. have used this technique with a Shirasu porous glass (SPG) membrane to formulate microparticles with lower size dispersion $[39,40]$. In order to overcome the low yield of this process, a premix membrane emulsification method was used to prepare monodispersed PLGA microparticles [41,42]. Alternatively to SPG membrane emulsification, an adjuvant strategy based on glass beads facilitated stirring, improved the homogeneity of PLGA microparticles produced by the emulsion/solvent evaporation method [43]. Although emulsion/solvent evaporation is often used to produce anti-TB drug loaded microparticles, it is labor intensive and not practicable for large production, which can limit translational strategies into therapies.

Inhalable microparticles and nano-in-microparticles were successfully obtained using the spray-drying (SD) technique [38,44-46], the most used technique for production of inhalable dry powders for antiTB delivery. SD is a simple, fast and versatile technique that can be used with different spray nozzles. For instance, rifampicin dihydrate microcrystals were coated with PLGA and/or PLA by SD with a three-fluid spray nozzle producing core-shell microcapsules with the appropriate aerodynamic properties to reach the alveoli and with controlled release properties not achieved by using only rifampicin dihydrate microcrystals $[14,15]$. Due to the existence of three independent channels, one for the core (drug), one for the shell (polymer) and the other for the 
Table 1

Summary of the techniques most often used in the preparation of polymeric microparticulate and nano-in-microparticulate systems for anti-TB pulmonary drug delivery.

\begin{tabular}{|c|c|c|c|c|}
\hline Technique & Brief explanation of working principle & Advantages of the process & Disadvantages of the process & Ref. \\
\hline $\begin{array}{c}\text { Emulsion/solvent } \\
\text { evaporation }\end{array}$ & $\begin{array}{l}\text { An organic solvent is used to dissolve the drug and polymer } \\
\text { followed by the addition of an aqueous solution containing a } \\
\text { dispersion stabilizer. The emulsion is sonicated followed by solvent } \\
\text { evaporation. }\end{array}$ & $\begin{array}{l}\text { Preserves the physicochemical } \\
\text { characteristics of the polymer }\end{array}$ & $\begin{array}{l}\text { Relatively wide size } \\
\text { distribution of microparticles } \\
\text { Use of organic solvents } \\
\text { Difficult to scale up } \\
\text { Two step process }\end{array}$ & {$[38,47]$} \\
\hline Spray-drying (SD) & $\begin{array}{l}\text { Transforms a feed in the fluid state into a dried particulate form by } \\
\text { spraying into a hot drying medium. }\end{array}$ & $\begin{array}{l}\text { Simple, fast, one-step, continuous } \\
\text { and reproducible production process } \\
\text { No final drying step } \\
\text { High versatility } \\
\text { Easy to scale up } \\
\text { Higher drug incorporation when } \\
\text { compared with other techniques }\end{array}$ & $\begin{array}{l}\text { Higher polydispersity when } \\
\text { compared with other } \\
\text { techniques } \\
\text { Expensive equipment and } \\
\text { operation conditions }\end{array}$ & {$[38,48]$} \\
\hline $\begin{array}{l}\text { Supercritical anti- } \\
\text { solvent }\end{array}$ & $\begin{array}{l}\text { The drug and polymer are dissolved in an organic solution and the } \\
\text { supercritical fluid }\left(\text { e.g. } \mathrm{SCO}_{2}\right) \text { acts as an anti-solvent promoting the } \\
\text { precipitation of the microparticles. }\end{array}$ & Single-step process & $\begin{array}{l}\text { Use of organic solvents } \\
\text { difficult to scale up } \\
\text { Expensive } \\
\text { Labour intensive }\end{array}$ & [49] \\
\hline $\begin{array}{l}\text { Anti-solvent } \\
\quad \text { Precipitation/SD }\end{array}$ & $\begin{array}{l}\text { Drop-wise addition of an anti-solvent (e.g. ethanol, diethyl ether)/ } \\
\text { drug solution to a polymer solution under rapid mixing. The drug } \\
\text { loaded nanoparticles are next spray dried with excipients to form } \\
\text { nanocomposites. }\end{array}$ & Low cost & $\begin{array}{l}\text { Two-step process } \\
\text { Use of organic solvents } \\
\text { Difficult to scale-up }\end{array}$ & [50] \\
\hline Ionotropic gelation/SD & $\begin{array}{l}\text { Drop-wise addition of cross-linking agent (e.g. TPP, } \mathrm{CaCl} 2 \text { ) into } \\
\text { polymer solution containing drug and under stirring. The drug } \\
\text { loaded micro- or nano- particles are next spray dried with or } \\
\text { without excipients to form microparticles or nanocomposites. }\end{array}$ & $\begin{array}{l}\text { Avoids the use of toxic reagents used } \\
\text { in the chemical cross-linking }\end{array}$ & $\begin{array}{l}\text { Two step process } \\
\text { Difficult to scale-up }\end{array}$ & {$[51,52]$} \\
\hline
\end{tabular}

Legend: $\mathrm{SCO}_{2}$ : supercritical carbon dioxide; SD: Spray-drying; TPP: tripolyphospate.

atomizing gas, the process can be done in a single step.

Nanocomposites containing rifampicin-PLGA nanoparticles and mannitol have also been prepared in a one-step approach using a fourfluid nozzle spray drier [53]. In this technique two liquid and two gas passages allowed the drug and the carrier to be prepared in different solvents avoiding the limitations associated to using a common solvent as in the traditional two fluid spray-drier.

Spray-drying can also be combined with techniques such as antisolvent precipitation and ionotropic gelation that are oriented to nanoparticle production, to fabricate nano-in-microparticulate systems. Inhalable guar gum nanoparticles were prepared using the precipitation technique with ethanol as anti-solvent [50]. Chitosan and alginate nanoparticles have been prepared by the ionotropic gelation, a method based on the complexation between oppositely charged species namely chitosan and tripolyphosphate anion or calcium cation and alginate $[51,52,54]$. The physical cross-linking by electrostatic interactions avoids toxic reagents used in the chemical cross-linking such as glutaraldeyde.

The supercritical technique has also been proposed for the production of inhalable polymeric microparticles. Patomchaiviwat et al. prepared rifampicin loaded PLA microparticles, in a size range suitable for lower airways deposition, using a supercritical anti-solvent process [49]. Supercritical anti-solvent is a quite recent and barely explored technique for anti-TB drug delivery. Although it is a single step process this technology is expensive and labor intensive and requires deeper investigation to assess its full potential for anti-TB drug delivery.

\subsection{Carrier systems oriented for macrophage targeting}

Phagocytosis of drug carrier systems by macrophages especially by infected macrophages is a very interesting approach leading to the internalization of these systems, and to an intracellular influx of the drug in the phagosome, where the bacilli reside, which could result in a more efficient anti-TB approach.

Among the physical properties, the size of the particulate carrier system is one of the most important characteristics affecting up-take via phagocytosis (Fig. 3) [55].

Phagocytosis ranged particles $(1-6 \mu \mathrm{m})$ enter the macrophage and can potentially deliver larger amounts of anti-TB drugs directly to the infection than oral or injected drug doses [56]. Particle shape also plays an important role in the up-take process as the local shape determines the initial contact with macrophages and their phagocytic fate [57]. Using shape-switching particles it was shown that elliptical disks have potential to mitigate phagocytosis, however when they switched their shape into spheres they were internalized by macrophages [58]. In relation to surface charge an increase up-take was observed when PLGA microparticles were coated with cationic polymer polyethylenimine relative to uncoated ones [43]. Furthermore, harder and non-porous particles are more efficiently taken up than soft and porous particles and hydrophobic and insoluble particles are more easily opsonized and this fact increases the probability of recognition by alveolar macrophages [55]. The chemical composition of the particle is also a significant feature for macrophage response. PLGA microparticles prepared with different proportions of lactide and glycolide and with different PLGA molecular weights result in different interactions with alveolar macrophages [59]. The presence of mannose moiety in the structure of polymers such as guar gum and mannan results in higher up-take by macrophages [51,60,61].

The carrier surface can be functionalized to incorporate ligands that target macrophage surface receptors in a process that is known as active targeting. Mannosylated gelatin microparticles have been developed to target macrophage mannose membrane receptor [62]. Other ligands including carbohydrate binding receptors such as galactose, $\beta$-glucan, $N$-acetylglycosamine and folic acid have been explored for macrophage targeting aiming at a wide range of diseases [63]. However, their potential towards therapeutic TB strategies requires further investigation.

\section{Emerging inhalable polymeric dry powders}

The main outcomes on recent works on dry powder microparticles and nano-in-microparticles for anti-TB drug delivery envisioning the improvement of inhalable administration of therapeutic drugs are compiled in Tables 2 and 3, respectively. 


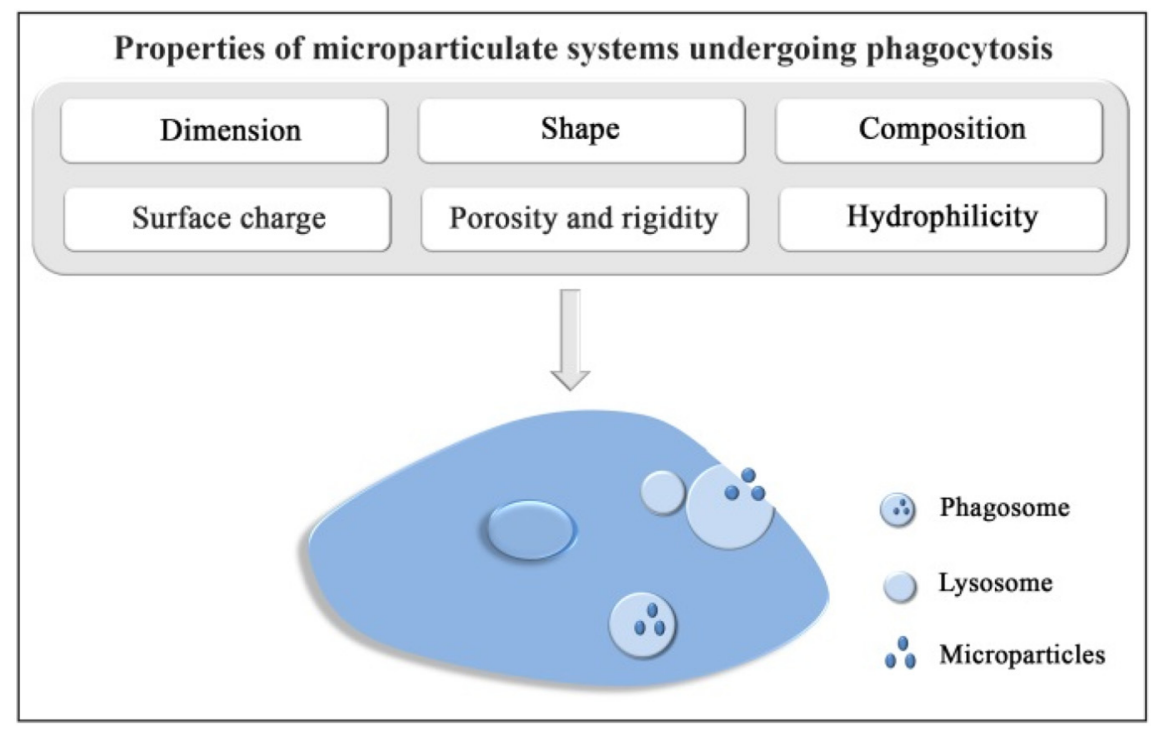

Fig. 3. Physico-chemical properties of microparticulate systems undergoing phagocytosis by alveolar macrophages.

\subsection{Polymeric microparticles}

\subsubsection{Poly(lactide-co-glycolide) (PLGA)}

The production of inhalable dry powder polymeric microparticles for pulmonary anti-TB drug delivery began with rifampicin loaded PLGA (R-PLGA) microparticles prepared by O'Hara and Hickey in 2000 [38]. In the meanwhile, the role of PLGA properties such as molecular weight and monomer composition have been studied to more accurately predict the loading efficiency and release rate of rifampicin [39]. Moreover, particles size [40], polymer content [65], $\mathrm{pH}$ of release medium [39] and presence of pulmonary surfactants in the release medium $[29,48]$ have also been assessed for their influence in rifampicin release profile.

R-PLGA microparticles have shown to be effectively phagocytosed by NR8383 macrophage cells and exerted a more potent bactericidal effect on Mycobacterium bovis Bacillus Calmette-Guérin infected NR8383 macrophage cells than a rifampicin solution [39,47,56,81-85]. These results were supported with in vivo studies, where the insufflation of R-PLGA microparticles to Wistar rats lead to 10 times greater amount of rifampicin in alveolar macrophages than a rifampicin insufflated powder [84]. Using Sprague-Dawley rats infected with Mtb Kurono strain as a model, intratracheal administration of R-PLGA microparticles was shown to be more efficient in killing the intracellular bacilli and on the prevention of granuloma formation in the lungs than a rifampicin powder [85]. The efficacy of inhalable R-PLGA microparticles was also assessed in Mtb (H37Rv)-infected guinea pigs by insufflation and/or nebulization into the lungs $[18,86]$. The bacterial burden and lung damage was significantly reduced in the presence of the microparticles and the effect of a single dose of R-PLGA microparticles was found to be comparable to that after treatment for 20 consecutive days with micronized rifampicin suspensions [87].

\subsubsection{Polylactide (PLA)}

Inhalable PLA microparticles were developed to incorporate both rifampicin and isoniazid as a promising carrier for anti-TB drug delivery [4]. These microparticles were up-taken by J774 murine macrophages and led to higher intracellular drug concentrations in comparison with equivalent amounts of drugs given in the culture medium. Mtb H37Ra infected J774 macrophages responded to rifampicin and isoniazid loaded PLA microparticles with a classical activation macrophages response not observed with drug solutions alone [45]. Additionally, rifampicin and isoniazid loaded PLA micropaticles administered to rats via intratracheal instillation and inhalation resulted in higher intracellular drug concentrations than oral and intracardiac injection delivery of soluble drugs [4]. These studies confirm that phagocytosis of particulate polymeric drug carriers results in the delivery of higher amounts of drugs to TB infected macrophages than those obtained from diffusive uptake of drugs dissolved in body fluids.

In another work by Muttil et al. rifabutin instead of rifampicin was loaded in inhalable PLA microparticles mainly because rifampicin is incompatible with isoniazid in organic solutions [5]. These rifabutin and isoniazid loaded PLA microparticles when administered to mice by inhalation or intra-tracheal instillation targeted macrophages as intracellular drug concentrations were found to be about 20 higher than serum concentrations. This approach also resulted in higher drug concentrations in macrophages than oral, intra-cardiac injection and even intra-tracheal instillation of free drug solutions $[5,88,89]$. Inhalation of rifabutin and isoniazid loaded PLA microparticles by Rhesus macaques also evidenced an efficient targeting of alveolar macrophages and lung tissue sparing non-target sites such as blood, liver and kidneys from the toxic drugs [90]. The ability of rifabutin and isoniazid loaded PLA microparticles to sustain higher levels of drugs in the cytosol of macrophages and for longer periods in comparison with soluble drugs highlight their efficacy in stimulating innate biological responses evoking a bactericidal action including induction of free radicals, changes in membrane potential and apoptosis [91-93].

\subsubsection{Poly-caprolactone (PCL)}

Although not so explored in inhaled anti-TB dry powders as PLGA and PLA, PCL has also been used to design microparticles suitable for pulmonary drug delivery [16,70]. In vitro and in vivo studies have shown that inhalable isoniazid loaded PCL (I-PCL) microparticles lead to a higher uptake as well as to a higher generation of mycobactericidal nitric oxide (NO) relative to isoniazid microparticles. This could be due to the hydrophobic nature of PCL favoring alveolar macrophages uptake and is an indication of the superior efficacy of PCL microparticles in comparison with isoniazid microparticles. As expected, intratracheal administration of I-PCL microparticles to rats lead to higher isoniazid accumulation in the lungs and less systemic drug availability and less hepatotoxicity effects over oral isoniazid administration. Thus, I-PCL microparticles prepared as inhalable dry powders may improve TB treatment efficacy and reduce hepatotoxicity which in turn will lead to a reduced period of treatment and improvement of patient compliance.

\subsubsection{Hydroxyl propyl methyl cellulose (HPMC)}

HPMC has been used as drug carrier [71,94] and inhalable dry 


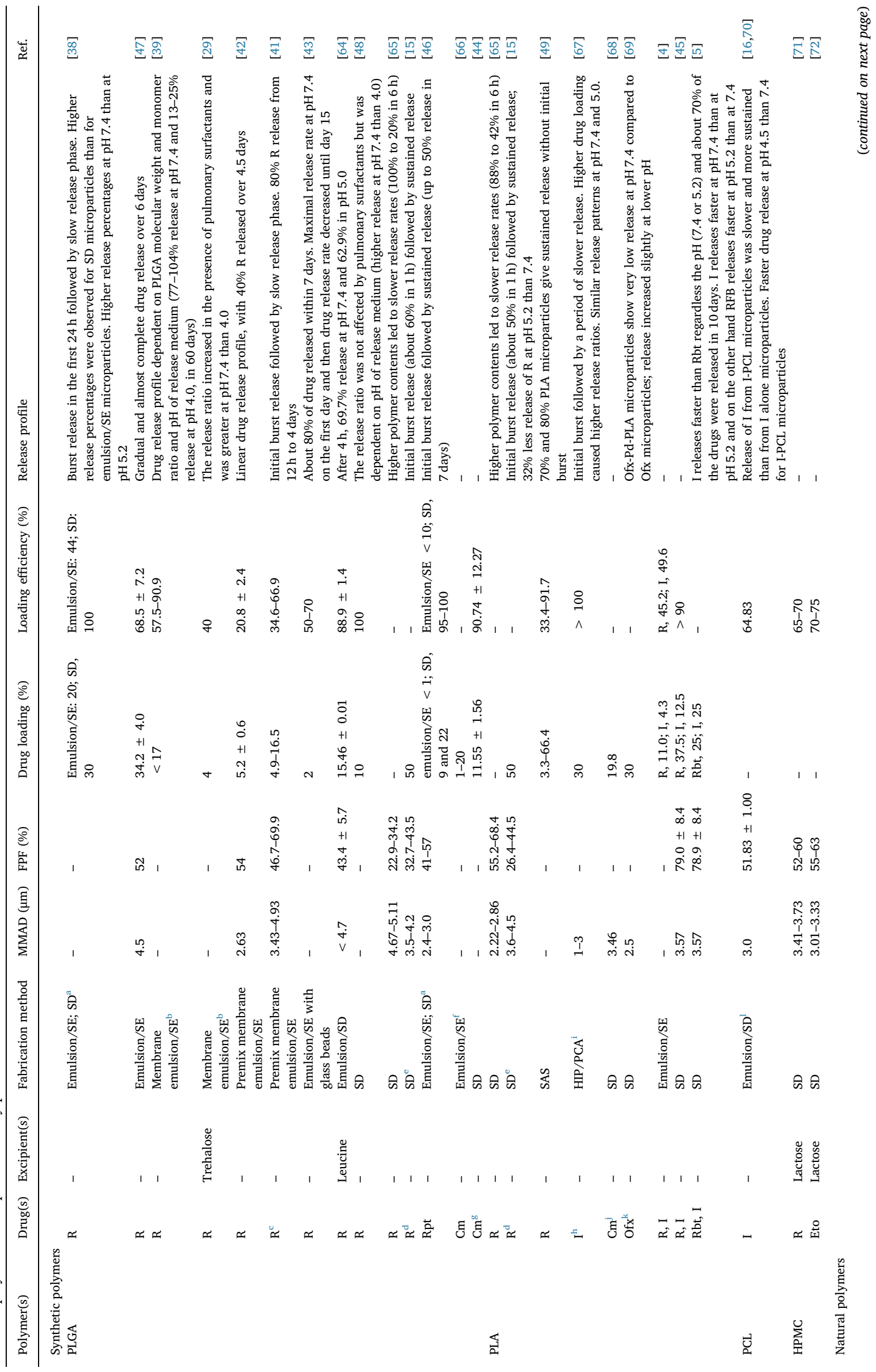




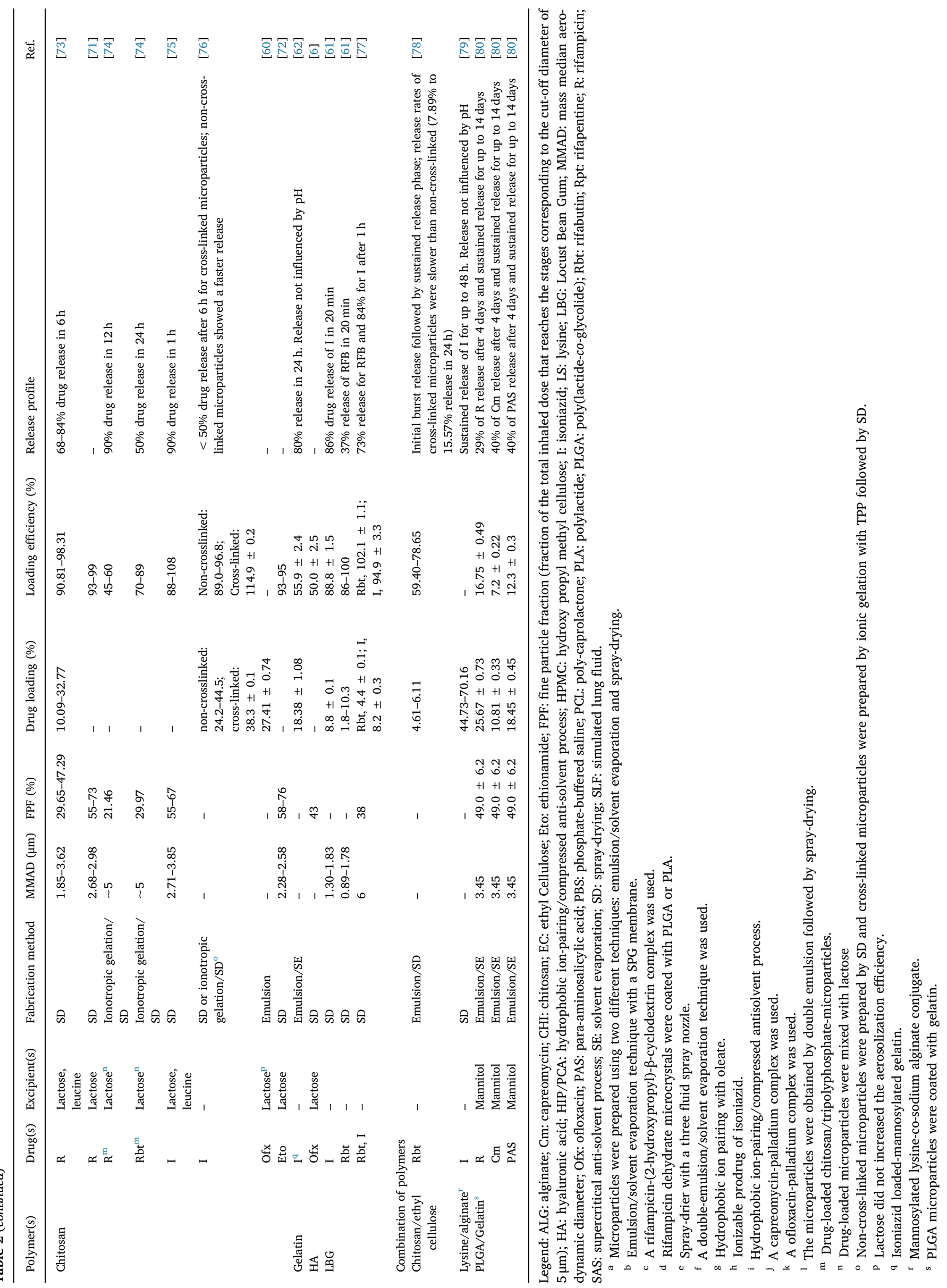




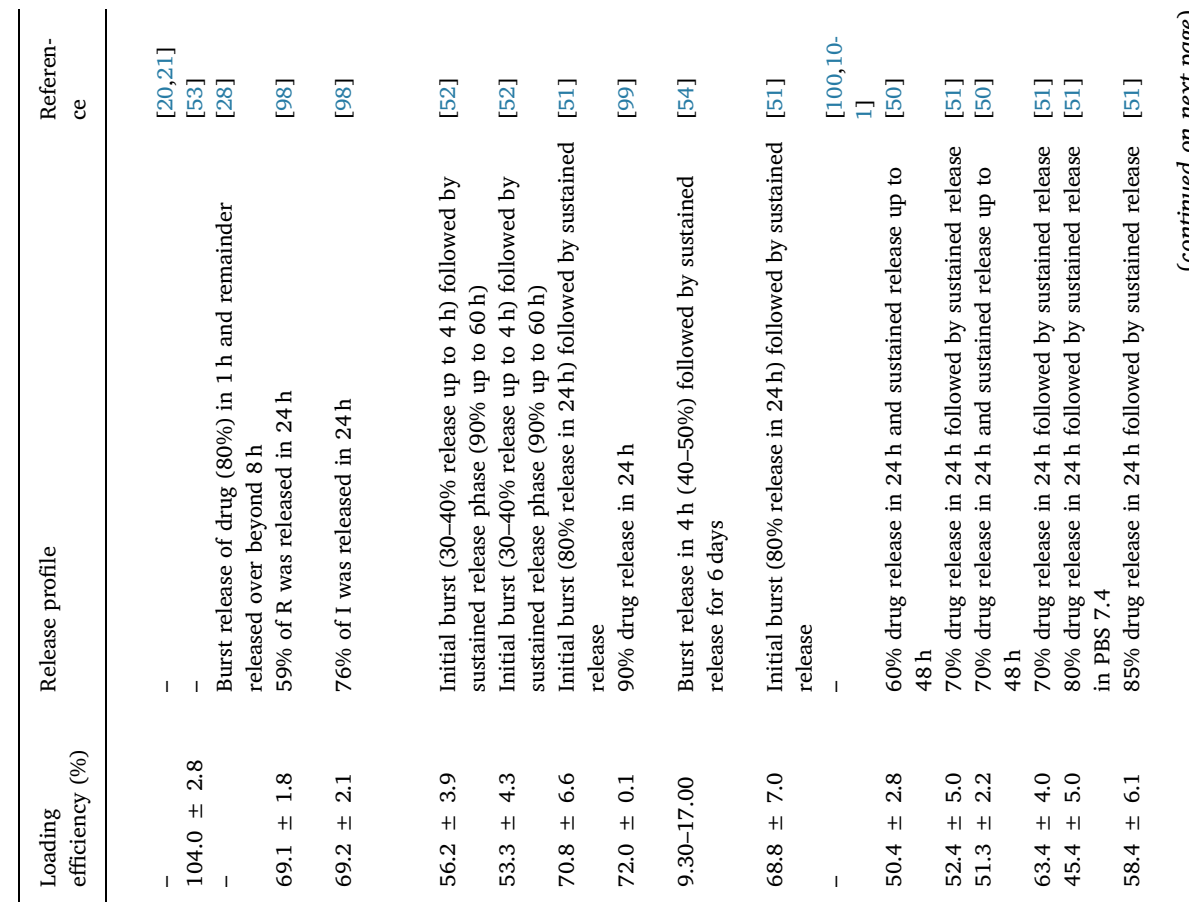

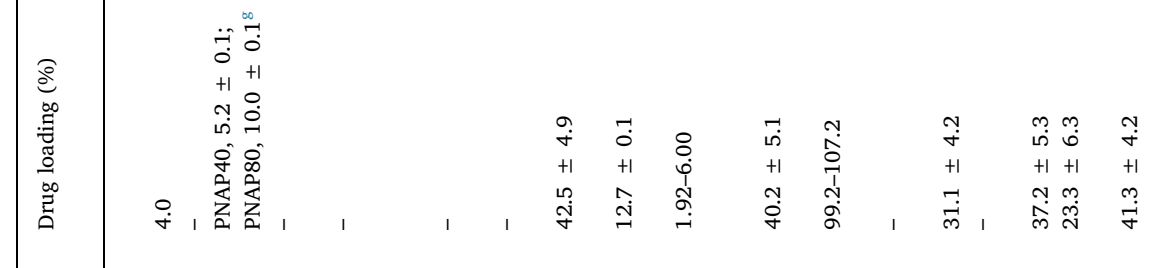

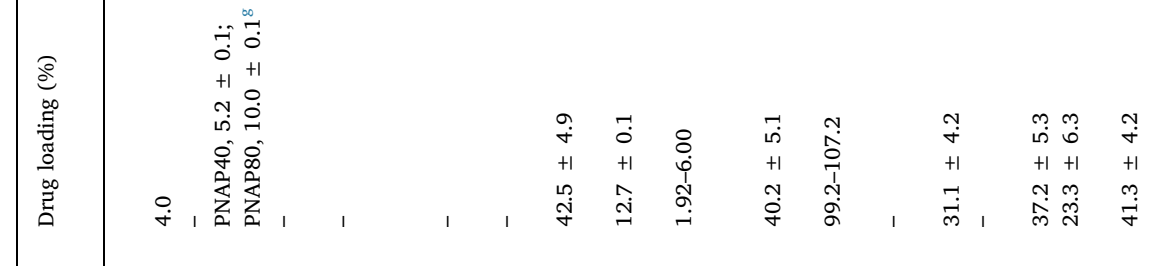

$\ddot{\vec{i}} \stackrel{\infty}{\stackrel{\infty}{m}}$

$+1+1$

ñ

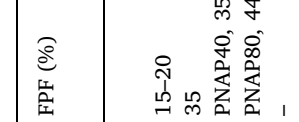

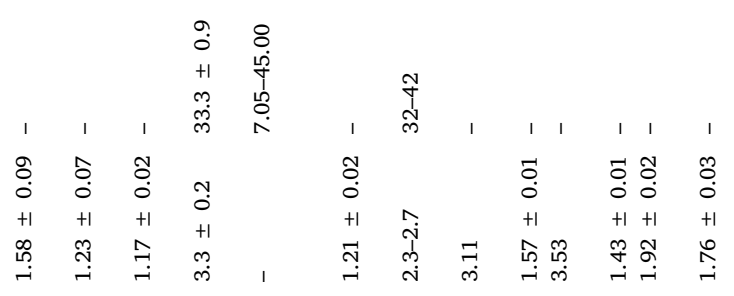

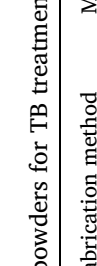

重

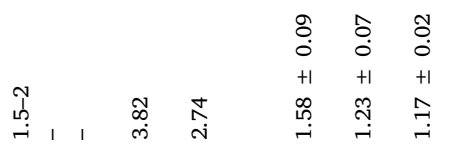

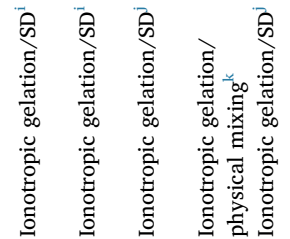

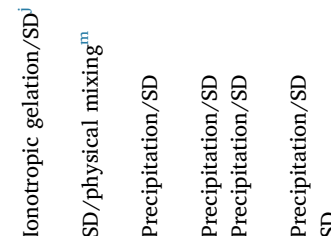

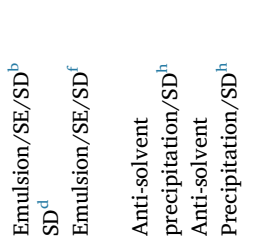

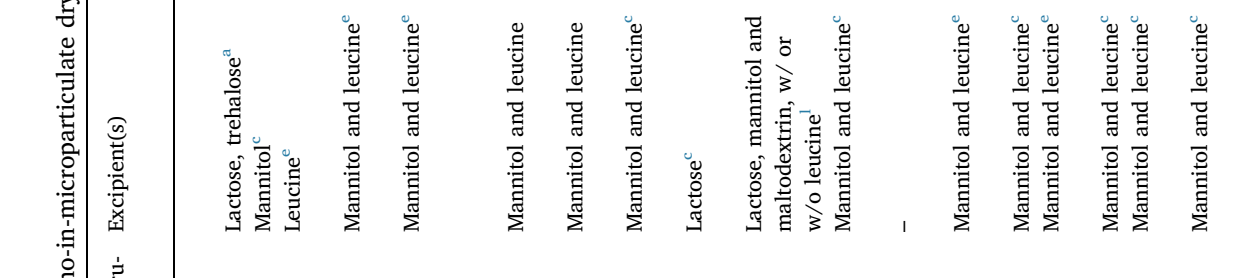

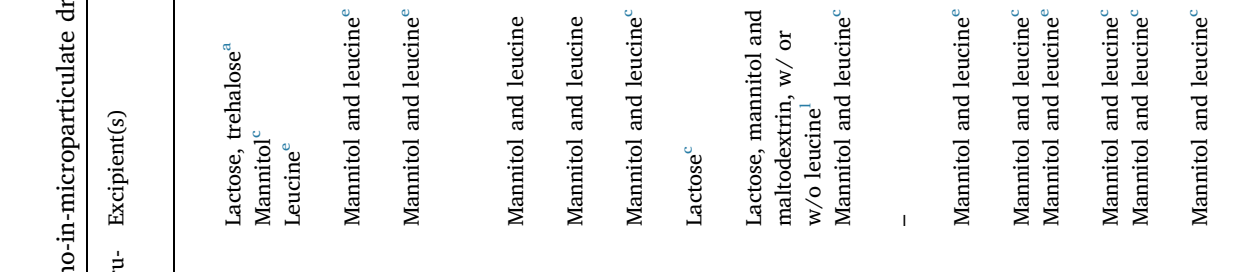

苞

से के के

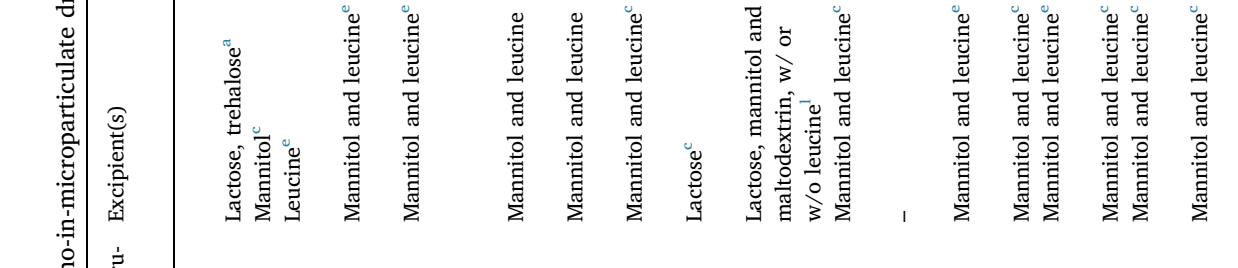

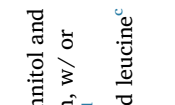

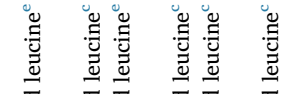

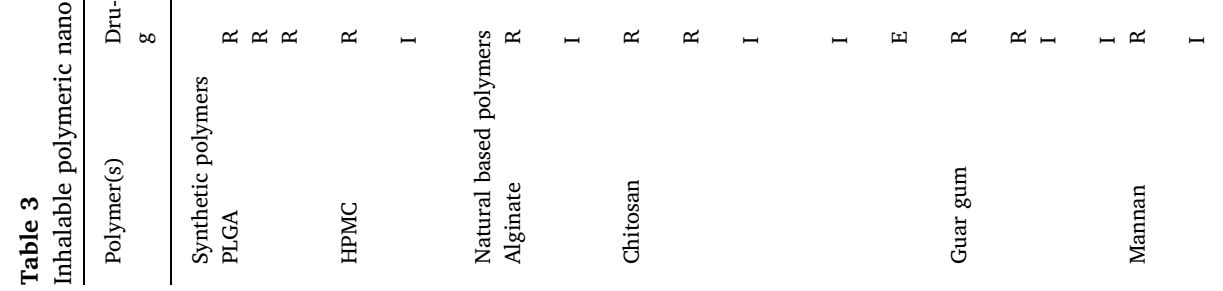




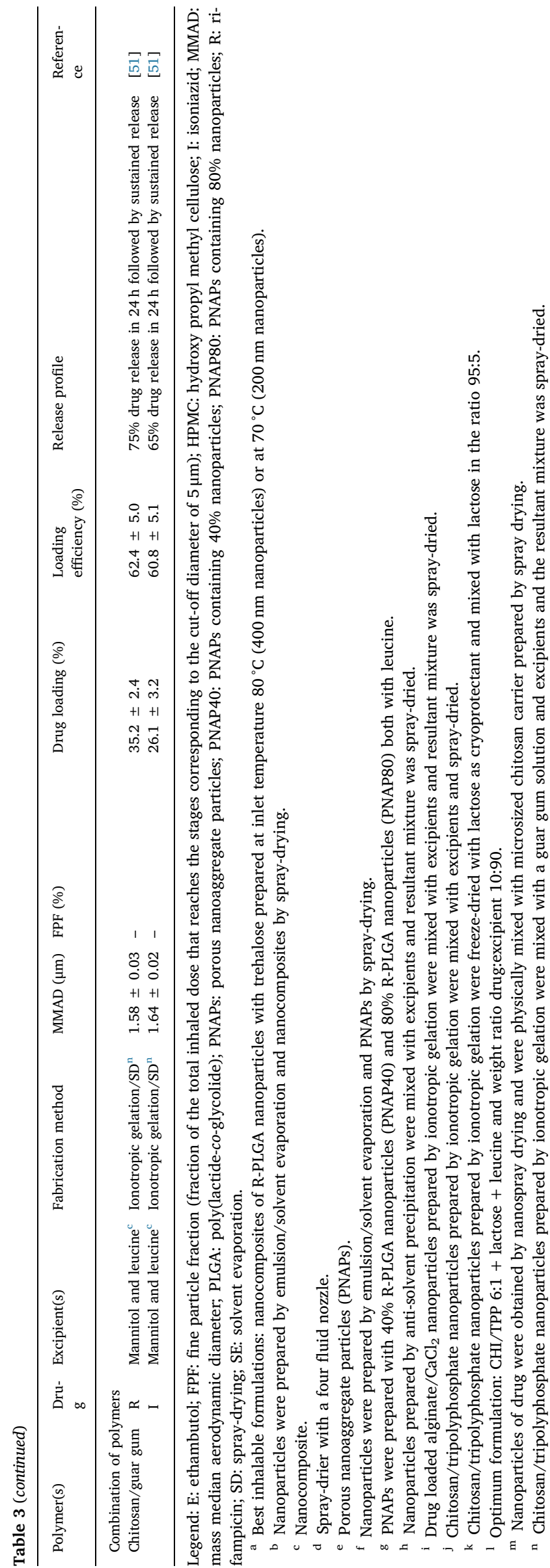

powder formulations have been prepared with rifampicin [71] and ethionamide [72], an anti-TB drug used in the treatment of MDR-TB. Although these studies suggest the suitability of HPMC formulations for reaching the lower airways, in vitro and in vivo studies are still needed to validate their application in TB treatment.

\subsubsection{Chitosan}

The mucoadhesive properties of chitosan are related to its cationic charge and makes chitosan more likely to adhere to the lungs and prolong drug release $[95,96]$. Moreover, chitosan particles are able to interact with the mannose receptors of macrophages, which results in increased phagocytosis [97]. Chitosan microparticles have been formulated with several anti-TB drugs including rifampicin [71,73,74], rifabutin [74], isoniazid [75,76], ofloxacin [60] and ethionamide [72] for pulmonary delivery. Cross-linked isoniazid loaded chitosan microparticles allowed a more sustained isoniazid release with $50 \%$ release in $6 \mathrm{~h}$ in comparison to non-crosslinked microparticles with over $50 \%$ released in $1 \mathrm{~h}$ [76]. Both non- and crosslinked microparticles can be of interest for TB treatment because in the first phase of the treatment, which is more intense, non-crosslinked microparticles may rapidly release isoniazid into the alveoli whereas cross-linked microparticles can be used in a second phase of the treatment (maintenance phase). Also the mucoadhesive properties of both non- and cross-linked chitosan microparticles were confirmed in vitro and in vivo, which can increase their time of residence in the lungs after inhalation. Moreover, both chitosan microparticles showed some toxic effect on murine peritoneal macrophages (J-774.1) which was microparticle concentration dependent $(0.01-1 \mathrm{mg} / \mathrm{mL})$ but no toxic effect on alveolar macrophages (AMJ2-C11). The activation of AMJ2-C11 cells with chitosan microparticles resulted in increased amounts of inflammatory cytokines: TNF- $\alpha$, IL-1 $\beta$, IL-6 in AMJ2-C11 cells in comparison to free isoniazid solubilized in culture medium.

As for other chitosan drug loaded microparticles, chitosan microparticles loaded with rifampicin and rifabutin showed to be less toxic to the lungs than free drug administration [74].

\subsubsection{Gelatin}

Although gelatin is not so deeply studied for pulmonary delivery, gelatin microparticles loaded with isoniazid have been produced to actively target alveolar macrophages [62]. As macrophages hold mannose membrane receptors, mannosylated gelatin microparticles exhibited significantly higher intracellular concentration of isoniazid than the non-mannosylated. Additionally, intratracheal administration of isoniazid loaded mannosylated gelatin microparticles to rats allowed achieving isoniazid therapeutic concentration in plasma. These results suggest the potential application of macrophage targeting in TB treatment that should be more deeply investigated.

\subsubsection{Hyaluronic acid (HA)}

Hyaluronic acid (HA) has also been explored for the development of microparticles for anti-TB pulmonary drug delivery. HA is a versatile polysaccharide involved in the assembly of the extracellular matrix (ECM) of numerous native tissues and has been applied in multiple clinical procedures. Ofloxacin loaded HA (Ofx-HA) microparticles have shown good aerosolization efficiency when combined with lactose [6]. Ofx-HA microparticles uptake by the alveolar macrophage cell line (RAW 264.7) lead to higher ofloxacin concentration than from ofloxacin microparticles or from an ofloxacin solution. Moreover, intratracheal administration of Ofx-HA microparticles to rats seems to be much more effective than intravenous or oral administration of an ofloxacin solution and intratracheal administration of ofloxacin microparticles for the treatment of TB.

\subsubsection{Locust Bean Gum (LBG)}

LBG is a polysaccharide composed of galactose and mannose units with potential application for macrophage targeting. Inhalable 
isoniazid and rifabutin loaded LBG microparticles were taken up by two macrophage cell lines: human macrophage-differentiated THP-1 cells and rat alveolar macrophages (NR 8383 cells) with high percentages of phagocytosis [61]. In another study by the same group LBG microparticles loaded with both drugs, isoniazid and rifabutin, thus meeting WHO requirements concerning combined TB therapy [77].

\subsubsection{Combination of polymers}

The idea of merging the advantageous properties of different polymers led to the development of microparticulate systems that result from the combination of polymers such as chitosan and ethyl cellulose [78]. Ethyl cellulose, which is a hydrophobic matrix carrier, was used for encapsulating and sustained release of the hydrophobic drug rifabutin, while chitosan was envisioned to provide bio-adhesion to the microparticles promoting pulmonary retention of the delivery system. The produced microparticles showed sustained release due to crosslinking with genipin and intratracheal administration to rats lead to prolonged pulmonary retention, thus maintaining drug concentrations at therapeutic level for at least 24 days.

Poly-lysine is a cationic polymer and the toxicity associated to its high charge density and high molecular weight restricts its use as a drug carrier system. Tiwari et al. coupled a low molecular weight lysine polymer with mannose in order to reduce the toxicity of the carrier system, having also a synergistic effect on the uptake by macrophages [62]. The lysine polymer was also conjugated with alginate to reduce the toxicity of the system, improve drug loading efficiency and for a sustained and controlled drug release. This mannosylated lysine conjugated to alginate (m-LS-co-ALG) natural based carrier system was used for pulmonary delivery of isoniazid. m-LS-co-ALG microparticles have no significant toxic effects in rat alveolar macrophages up to a concentration as high as $20 \mathrm{mg} / \mathrm{mL}$. Isonizaid loaded m-LS-co-ALG microparticles, intratracheally administered to rats, were effectively up-taken by alveolar macrophages and prolonged the presence of isoniazid in the blood.

The most studied polymers for microparticle production for inhalable delivery are PLGA and PLA although natural polymers such as chitosan have gained great interest in recent years due to their availability, cost-effectiveness and resemblance to biopolymers from living tissues. Despite chitosan being an attractive candidate for inhalable drug delivery other natural sources should be considered and their applicability explored. Biopolymers as collagen or chondroitin sulfate are natural materials used for particle fabrication in biomedical applications that could be transposed and investigated for TB approaches.

Different polymers have shown promising results in the design and loading of anti-TB drugs in clinical use. The incorporation of anti-TB drugs in polymer based carriers often increases the drug solubility, allows the protection of the drug before reaching the target site and allows modulating the release profile of the drugs assisting a sustained release.

With very few exceptions, studies report microparticulate systems incorporating first line drugs. This approach is quite useful to improve treatments efficiency by reducing the drug dosage (without prejudice to their effectiveness), reduction of systemic toxicity, reducing the regimen period and thus contributing to the eradication of the disease. Nevertheless, the development of polymeric dry powder delivery systems is also envisioned to contribute to anti-TB therapies when the treatment fails. Second line drugs as capreomycin and ofloxacin, which are typically more toxic that first line drugs, could have their dosage reduced and the parental administration replaced by the pulmonary route favoring stability, storage and shelf life. This approach would also favor patient compliance to the treatment and likely increase the success rate in drug resistant-TB strategies.

\subsection{Polymeric nano-in-microparticles}

\subsubsection{Poly(lactide-co-glycolide) (PLGA)}

Rifampicin loaded PLGA nanocomposites have been produced by Tomoda et al. to be used as an inhalation system [20,21]. The influence of the excipient, size of primary nanoparticles, weight ratio nanoparticle:excipient and inlet temperature of the spray dryer on the aerodynamic and decomposition properties of the nanocomposites were investigated. Nanoparticles with dimensions of $200 \mathrm{~nm}$ and $400 \mathrm{~nm}$, combined with trehalose at the inlet temperatures of $70^{\circ} \mathrm{C}$ and $80^{\circ} \mathrm{C}$, respectively, were considered the most promising ones for pulmonary delivery. These results were independent from the weight ratio nanoparticle:excipient.

Rifampicin loaded PLGA nanocomposites prepared with mannitol (R-PLGA/MAN) were readily dispersed in culture medium and dissociate to nanoparticles which are not so efficiently in vitro up-taken by NR8383 cells (13.5\%) as micro-sized rifampicin loaded PLGA particles (47.1\%) [53]. Conversely, intra-tracheal administration of R-PLGA/ MAN nanocomposites to rats lead to a higher rifampicin uptake (about 9.3\% after $4 \mathrm{~h}$ ) by alveolar macrophages in comparison with R-PLGA and R/MAN microparticles which was explained by the difficulty to clear the nanoparticles from the lungs.

Intratracheal insufflation of rifampicin-PLGA PNAPs formulations to guinea pigs led to a prolonged presence of rifampicin in the lungs up to $8 \mathrm{~h}$ whereas insufflated rifampicin porous particles or rifampicin given intravenously or orally showed low or no levels of rifampicin in the lungs [28].

\subsubsection{Hydroxy propyl methyl cellulose (HPMC)}

PNAPs of HPMC loaded with rifampicin and isoniazid were prepared with mannitol and leucine to improve yield and aerodynamic properties of the powders [98]. $24 \mathrm{~h}$ after insufflation of the developed PNAPs in rats, approximately $25 \%$ of drug was still in the lungs while in the case of plain drug administration no drug was detected. Moreover, organ distribution studies showed a lower level of drug from the developed PNAPs in the liver and kidney in comparison with plain drug due to the controlled drug release from the PNAPs formulations.

\subsubsection{Alginate}

Alginate nanocomposites have been developed for pulmonary delivery of rifampicin and isoniazid [52]. The minimum inhibitory concentration (MIC) of rifampicin $(0.2 \pm 0.01 \mu \mathrm{g} / \mathrm{mL})$ and isoniazid $(0.1 \pm 0.01 \mu \mathrm{g} / \mathrm{mL})$ loaded alginate nanocomposites against Mtb was approximately 3-4 times lower than that of free rifampicin $(0.5 \pm 0.01 \mu \mathrm{g} / \mathrm{mL})$ and isoniazid $(0.4 \pm 0.01 \mu \mathrm{g} / \mathrm{mL})$ solutions. Developed formulations insufflated to mice could be detected in the lungs for up to $8 \mathrm{~h}$ ensuring a localized and controlled drug delivery and exhibited a higher rate and extent of drug uptake by lungs than by liver, spleen and kidney.

\subsubsection{Chitosan}

Chitosan nanocomposites have been prepared for anti-TB pulmonary drug delivery. Inhalable rifampicin loaded chitosan nanocomposites have shown negligible in vitro toxicity on murine J774 macrophage cells [99]. Inhalation of the developed formulation by rats lead to a higher maximum drug concentration and a higher residence time in the lungs (up to $24 \mathrm{~h}$ ) in comparison with a rifampicin commercial powder, explained by the sustained release of rifampicin from the developed formulation. Moreover, only mild changes were found in the pathology of treated lungs from the developed formulation unlike a commercial rifampicin solution given orally that showed severe toxicity.

\subsubsection{Guar gum}

The presence of the mannose moiety in guar gum imparts a great affinity towards macrophage mannan receptors increasing macrophage cells up-take [51]. Rifampicin and Isoniazid loaded guar gum PNAPs insufflated to rats revealed a greater amount of drug in the lungs in comparison with the liver and kidneys [50]. Moreover, unlike plain drug administration, about $15 \%$ of the drugs from the developed formulations were still detected in the lungs after $24 \mathrm{~h}$, which was ascribed 
to the mucoadhesive properties of guar gum and the ability to control the release of the drugs from the developed systems.

\subsubsection{Mannan}

Mannan is a linear polysaccharide of mannose subunits which could favor specific recognition by macrophages and potentiate phagocytosis. Inhalable rifampicin and isoniazid loaded mannan nanocomposites have demonstrated an excellent uptake by J-774 macrophage cells and a MIC about 15 times lower than those of rifampicin and isoniazid solutions [51]. However, when insufflated to Mtb infected mice they exhibited a low lung residence time due to their intrinsic solubility.

\subsubsection{Combination of polymers}

Goyal et al. prepared nanocomposites made of different polymers namely chitosan, guar gum, mannan and guar gum coated chitosan nanocomposites [51]. Guar gum coated chitosan nanocomposites were shown to be the most promising for pulmonary anti-TB drug delivery. Guar gum coated rifampicin and isoniazid loaded chitosan nanocomposites insufflated to Mtb infected mice showed a prolonged residence of the drug in the lungs thereby improving the therapeutics utility and resulted in almost 5 -fold reduction of the number of bacilli as compared to the untreated control group 45 days post administration [51].

Although the nano-in-microparticles produced are promising for drug delivery these particles are still less studied than microparticles for TB treatment. The anti-TB drug loading in nano-in-microparticles is increased over microparticles due to a greater surface area/volume ratio of nanoparticles. Nano-in-microparticles re-disperse into the elementary nanoparticles in the lung lining fluid and due to their dimensions they are not cleared as fast as the microparticles, which may lead to an increased presence in the lungs leading to a higher drug up-take [53]. However, as referred in Section 2.5, the production of nano-inmicroparticles relies on a combination of techniques and a multi-step production, which is labor intensive and difficult to scale up.

Overall, polymeric systems either in the form of microparticles or as nano-in-microparticles have been developed with improved functionalities to efficiently transport and release the drug to the lung alveoli and to target a resident population of macrophages. Independently of the polymer systems and associated specificities, the advantage of using polymeric dry powders as drug loading microparticulate system over direct drug administration to the lungs is evident for pulmonary delivery and towards the treatment of TB.

It is important, however, to establish standardized models, both in vitro and in vivo, that enable to more accurately compare the effectiveness and therapeutic potential of the drug loaded particulate systems developed. It is critical to study these systems in models that mimic the transport along the human airways, considering real therapeutic dosages given to human patients and the challenges involved in the mechanisms for drug release and particle uptake in macrophages in order to validate their application in clinics.

\subsection{Targeting host cell responses}

Mtb has co-evolved with humans for thousands of years and has developed several strategies to prevent elimination by its host. Hostmediated responses, such as activation of protective inflammatory mediators, including cytokines and chemokines that induce natural microbicidal pathways and recruit white blood cells to the infection site, may provide an efficient mean to effectively eliminate Mtb and targeting host responses has been recently explored for TB treatment. However, a considerable disadvantage over bacteria-oriented approaches is the toxicity to the host cells, which can be overcome to a certain extent using carrier systems.

Verma et al. explored alveolar macrophage response to NO using PLGA microparticles containing NO donors (isosorbide mononitrate, sodium nitroprusside or diethylenetriamine nitric oxide adduct) [102].
NO is known to possess mycobactericidal properties and indeed it is one of the most important macrophage microbicidal mechanisms. The addition of anti-TB drugs rifabutin and isoniazid further increase the efficiency of the developed system [103]. Gupta et al. produced inhalable PLGA microparticles for targeting rapamycin, which is an inducer of autophagy, to alveolar macrophages [104]. The PLGA microparticles containing rapamycin were less toxic to host cells and more effective in inducing the clearing of intracellular Mtb, as compared to rapamycin in the free form. The efficiency of this system was also found to increase in conjunction with inhalable rifabutin and isoniazid [105]. Other systems of inhalable particles containing nitazoxanide, which has moderate mycobactericidal activity and is also an inducer of autophagy in mammalian cells, were incorporated alone or in combination with rifabutin and isoniazid agents [106]. The combination of these drugs incorporated in the polymeric microparticles cleared Mtb H37Rv from lungs and spleen of infected mice and restored tissue architecture in higher extent than rifabutin-isoniazid particles.

\subsection{Magnetic nanoparticles for anti-TB drug therapies}

Magnetic nanoparticles (MNPs) have shown potential for a wide range of biomedical applications, including biosensing, imaging and targeted drug delivery, as MNPs can be manipulated under the influence of a magnetic field $[107,108]$. MNPs have already been approved for clinical use as contrast agents in magnetic resonance imaging (MRI) allowing the diagnostic of several diseases. Additionally, MNPs can be oriented and concentrated towards a given tissue location via an external magnetic field actuation allowing a more efficient treatment and minimizing side effects. Magnetized aerosols comprising superparamagnetic iron oxide nanoparticles (SPIONs) have been studied for pulmonary delivery of drugs and genes to treat diseases of the deeper airways $[109,110]$. These nanomagnetosols have shown potential to be in vivo controlled and spatially guided in the lungs using an external magnetic gradient field.

Despite the promising outcomes of MNPs for pulmonary delivery applications, few studies refer their use in inhalable dry powders for TB treatment strategies. MNPs can be incorporated within microparticulate systems and be part of a sophisticated imaging and guiding system for pulmonary drug delivery purposes. Tewes et al. fabricated SPIONsloaded Trojan microparticles for targeting bacterial infectious pulmonary foci upon loading with the appropriate drug [111]. This preliminary study showed that the developed microparticles have good aerodynamic properties and their deposition was found to be highly sensitive to the actuation of an external magnetic field. Moreover, the drug release profile can be modulated according to the treatment requirements with the application of an external stationary or alternating magnetic fields, contributing for improved treatment efficiency [112]. In a recent paper gelatin-based microparticles containing SPIONs were proposed as inhalable dry-powder carriers for a candidate anti-TB drug to be released inside alveolar macrophages [113]. The actuation of an external alternating magnetic field was shown to remotely trigger and modulate the anti-TB drug release, which could contribute for improved strategies for TB treatment.

\section{Conclusions and future perspectives}

Tuberculosis is a worldwide disseminated disease with potential to be treated and ultimately eradicated. Current therapeutic regimens show limitations than can be overcome with more sophisticated and oriented approaches, such as using inhalable polymeric dry powders for anti-TB drug delivery. These systems are quite versatile and growing evidence suggests that they are also more efficient in the drug targeting macrophages than oral or injected drug administration and even drug free inhalation. Moreover, dry powders show promising outcomes for the treatment of lung diseases and with impact in the quality of life of patients. The fact that they are easily stored, cost-effective and simple 
to administer are attractive features for up-scaling and commercialization. Magnetic particles can add clinical significance to the system as a valuable tool to guide the system to a given location and, simultaneously provide a non-invasive real-time monitoring system during the follow-up of patients after drug administration.

Moreover, the ability to target phagocytosed bacilli or the cells involved in Mtb clearance is a considerable advantage to be deeper investigated aiming at personalized strategies, considering each patient individual needs and orient the most suitable treatment not only in terms of drug selection but also targeting a specific location (either alveoli niche, alveoli macrophage, Mtb bacilli or the granuloma) to better suit the patient needs.

In the future, pulmonary delivery systems can eventually combine the administration of one or multiple pharmaceutical agents to induce different yet synergistic responses for instance, microbiocidal effect over Mtb and simultaneously target alveoli macrophages towards a more effective and shorter treatment regimen.

The challenge in upcoming years also resides in fine tuning inhalable dry powder carriers focusing on their multifactorial nature and final application, accompanying the progress of new anti-TB formulations to meet individual needs with simple yet efficient drug delivery systems to be routinely used in clinical therapies.

\section{Acknowledgments}

The authors wish to acknowledge the financial support from the Portuguese Foundation for Science and Technology (FCT) for the postdoctoral grant of M.S.M (SFRH/BPD/110868/2015), M.T.R (SFRH/ BPD/111729/2015) and R.M.A.D (SFRH/BPD/112459/2015), FCT grant of E.T. (IF/01390/2014) and Recognize project (UTAP-ICDT/ CTM-BIO/0023/2014). This article is also a result of the project "Accelerating tissue engineering and personalized medicine discoveries by the integration of key enabling nanotechonologies, marine-derived biomaterials and stem cells", supported by Norte Portugal Regional Operational Programme (NORTE 2020), under the PORTUGAL 2020 Partnership Agreement, through the European Regional Development Fund (ERDF). The authors acknowledge the financial support from the European Union Framework Programme for Research and Innovation HORIZON 2020, under the TEAMING Grant agreement No 739572 The Discoveries CTR.

\section{References}

[1] S.H.E. Kaufmann, How can immunology contribute to the control of tuberculosis? Nat. Rev. Immunol. 1 (2001) 20-30.

[2] WHO, World Health Organization, Global Tuberculosis Report 2017, (2017).

[3] http://www.who.int/en/news-room/fact-sheets/detail/tuberculosis.

[4] R. Sharma, D. Saxena, A.K. Dwivedi, A. Misra, Inhalable microparticles containing drug combinations to target alveolar macrophages for treatment of pulmonary tuberculosis, Pharm. Res. 18 (2001) 1405-1410.

[5] P. Muttil, J. Kaur, K. Kumar, A.B. Yadav, R. Sharma, A. Misra, Inhalable microparticles containing large payload of anti-tuberculosis drugs, Eur. J. Pharm. Biopharm. 32 (2007) 140-150.

[6] S.M. Hwang, D.D. Kim, S.J. Chung, C.K. Shim, Delivery of ofloxacin to the lung and alveolar macrophages via hyaluronan microspheres for the treatment of tuberculosis, J. Control. Release 129 (2008) 100-106.

[7] D. Dube, G.P. Agrawal, S.P. Vyas, Tuberculosis: from molecular pathogenesis to effective drug carrier design, Drug Discov. Today 17 (2012) 760-773.

[8] A.S. Dharmadhikari, M. Kabadi, B. Gerety, A.J. Hickey, P.B. Fourie, E. Nardell, Phase I, single-dose, dose-escalating study of inhaled dry powder capreomycin: a new approach to therapy of drug-resistant tuberculosis, Antimicrob. Agents Chemother. 57 (2013) 2613-2619.

[9] J.G.Y. Chan, A.S. Tyne, A. Pang, A.J. McLachlan, V. Perera, J.C.Y. Chan, W.J. Britton, H.K. Chan, C.C. Duke, P.M. Young, D. Traini, Murine pharmacokinetics of rifapentine delivered as an inhalable dry powder, Int. J. Antimicrob. Agents 45 (2015) 319-323.

[10] J.G.Y. Chan, C.C. Duke, H.X. Ong, J.C.Y. Chan, A.S. Tyne, H.-K. Chan, W.J. Britton, P.M. Young, D. Traini, A novel inhalable form of rifapentine, J. Pharm. Sci. 103 (2014) 1411-1421.

[11] L. Garcia Contreras, J. Sung, M. Ibrahim, K. Elbert, D. Edwards, A. Hickey, Pharmacokinetics of inhaled rifampicin porous particles for tuberculosis treat ment: insight into rifampicin absorption from the lungs of guinea pigs, Mol.
Pharm. 12 (2015) 2642-2650.

[12] R. Parikh, L. Patel, S. Dalwadi, Microparticles of rifampicin: comparison of pulmonary route with oral route for drug uptake by alveolar macrophages, phagocytosis activity and toxicity study in albino rats, Drug Deliv. 21 (2014) 406-411.

[13] N.R. Labiris, M.B. Dolovich, Pulmonary drug delivery. Part I: physiological factors affecting therapeutic effectiveness of aerosolized medications, Br. J. Clin. Pharmacol. 56 (2003) 588-599.

[14] Y.-J. Son, J.T. McConville, A new respirable form of rifampicin, Eur. J. Pharm. Biopharm. 78 (2011) 366-376.

[15] Y.J. Son, J.T. McConville, Preparation of sustained release rifampicin microparticles for inhalation, J. Pharm. Pharmacol. 64 (2012) 1291-1302.

[16] R. Parikh, S. Dalwadi, Preparation and characterization of controlled release poly$\varepsilon$-caprolactone microparticles of isoniazid for drug delivery through pulmonary route, Powder Technol. 264 (2014) 158-165.

[17] M.M. Gaspar, A. Cruz, A.F. Penha, J. Reymão, A.C. Sousa, C.V. Eleutério, S.A. Domingues, A.G. Fraga, A.L. Filho, M.E.M. Cruz, J. Pedrosa, Rifabutin encapsulated in liposomes exhibits increased therapeutic activity in a model of disseminated tuberculosis, Int. J. Antimicrob. Agents 31 (2008) 37-45.

[18] S. Suarez, P. O'Hara, M. Kazantseva, C.E. Newcomer, R. Hopfer, D.N. McMurray, A.J. Hickey, Airways delivery of rifampicin microparticles for the treatment of tuberculosis, J. Antimicrob. Chemother. 48 (2001) 431-434.

[19] N.V. Satheesh Madhav, S. Kala, Review on microparticulate drug delivery system, Int. J. PharmTech Res. 3 (2011) 1242-1254.

[20] K. Tomoda, T. Ohkoshi, T. Nakajima, K. Makino, Preparation and properties of inhalable nanocomposite particles: effects of the size, weight ratio of the primary nanoparticles in nanocomposite particles and temperature at a spray-dryer inlet upon properties of nanocomposite particles, Colloids Surf. B: Biointerfaces 64 (2008) 70-76.

[21] K. Tomoda, T. Ohkoshi, Y. Kawai, M. Nishiwaki, T. Nakajima, K. Makino, Preparation and properties of inhalable nanocomposite particles: effects of the temperature at a spray-dryer inlet upon the properties of particles, Colloids Surf. B: Biointerfaces 61 (2008) 138-144.

[22] L. Gradon, T.R. Sosnowski, Formation of particles for dry powder inhalers, Adv. Powder Technol. 25 (2014) 43-55.

[23] B.K. Nanjwade, S.A. Adichwal, K.R. Gaikwad, K.A. Parikh, F.V. Manvi, Pulmonary drug delivery: novel pharmaceutical technologies breathe new life into the lungs, PDA J. Pharm. Sci. Technol. 65 (2011) 513-534.

[24] G. Pilcer, K. Amighi, Formulation strategy and use of excipients in pulmonary drug delivery, Int. J. Pharm. 392 (2010) 1-19.

[25] T. Sethi, A. Agrawal, Structure and function of the tuberculous lung: considerations for inhaled therapies, Tuberculosis 91 (2011) 67-70.

[26] N.P. Mortensen, P. Durham, A.J. Hickey, The role of particle physico-chemical properties in pulmonary drug delivery for tuberculosis therapy, J. Microencapsul 31 (2014) 785-795.

[27] D.E. Goodman, E. Israel, M. Rosenberg, R. Johnston, S.T. Weiss, J.M. Drazen, The influence of age, diagnosis, and gender on proper use of metered-dose inhalers, Am. J. Respir. Crit. Care Med. 150 (1994) 1256-1261.

[28] J.C. Sung, D.J. Padilla, L. Garcia-Contreras, J.L. Verberkmoes, D. Durbin, C.A. Peloquin, K.J. Elbert, A.J. Hickey, D.A. Edwards, Formulation and pharmacokinetics of self-assembled rifampicin nanoparticle systems for pulmonary delivery, Pharm. Res. 26 (2009) 1847-1855.

[29] K. Tomoda, K. Makino, Effects of lung surfactants on rifampicin release rate from monodisperse rifampicin-loaded PLGA microspheres, Colloids Surf. B: Biointerfaces 55 (2007) 115-124.

[30] A. Grobler, Z. Perez Sierra, H.J. Viljoen, Modeling nanoparticle delivery of TB drugs to granulomas, J. Theor. Biol. 388 (2016) 85-95.

[31] S.B. Walters, J. Kieckbusch, G. Nagalingam, A. Swain, S.L. Latham, G.E.R. Grau, W.J. Britton, V. Combes, B.M. Saunders, Microparticles from mycobacteria-infected macrophages promote inflammation and cellular migration, J. Immunol 190 (2013) 669-677.

[32] D.-D. Pham, E. Fattal, N. Tsapis, Pulmonary drug delivery systems for tuberculosis treatment, Int. J. Pharm. 478 (2015) 517-529.

[33] L. Willis, D. Hayes, H.M. Mansour, Therapeutic liposomal dry powder inhalation aerosols for targeted lung delivery, Lung 190 (2012) 251-262.

[34] W.B. Liechty, D.R. Kryscio, B.V. Slaughter, N.A. Peppas, Polymers for drug delivery systems, Annual Review of Chemical and Biomolecular Engineering, 12010 pp. 149-173.

[35] B. Patel, V. Gupta, F. Ahsan, PEG-PLGA based large porous particles for pulmonary delivery of a highly soluble drug, low molecular weight heparin, J. Control. Release 162 (2012) 310-320.

[36] U. Francesca, d.A. Ivana, M. Agnese, L.R.M. I., Q. Fabiana, Engineered PLGA nanoand micro-carriers for pulmonary delivery: challenges and promises, J. Pharm. Pharmacol. 64 (2012) 1217-1235.

[37] M. Beck-Broichsitter, K. Stoisiek, A. Bohr, L. Aragão-Santiago, T. Gessler, W. Seeger, T. Kissel, Potential of the isolated lung technique for the examination of sildenafil absorption from lung-delivered poly(lactide-co-glycolide) microparticles, J. Control. Release 226 (2016) 15-20.

[38] P. O'Hara, A.J. Hickey, Respirable PLGA microspheres containing rifampicin for the treatment of tuberculosis: manufacture and characterization, Pharm. Res. 17 (2000) 955-961.

[39] K. Makino, T. Nakajima, M. Shikamura, F. Ito, S. Ando, C. Kochi, H. Inagawa, G. Soma, H. Terada, Efficient intracellular delivery of rifampicin to alveolar macrophages using rifampicin-loaded PLGA microspheres: effects of molecular weight and composition of PLGA on release of rifampicin, Colloids Surf. B: Biointerfaces 36 (2004) 35-42.

[40] F. Ito, K. Makino, Preparation and properties of monodispersed rifampicin-loaded 
poly(lactide-co-glycolide) microspheres, Colloids Surf. B: Biointerfaces 39 (2004) $17-21$.

[41] T.V. Doan, W. Couet, J.C. Olivier, Formulation and in vitro characterization of inhalable rifampicin-loaded PLGA microspheres for sustained lung delivery, Int. J. Pharm. 414 (2011) 112-117.

[42] T.V. Doan, J.C. Olivier, Preparation of rifampicin-loaded PLGA microspheres for lung delivery as aerosol by premix membrane homogenization, Int. J. Pharm. 382 (2009) 61-66.

[43] Z. Liu, X. Li, B. Xiu, C. Duan, J. Li, X. Zhang, X. Yang, W. Dai, H. Johnson, H. Zhang, X. Feng, A novel and simple preparative method for uniform-sized PLGA microspheres: preliminary application in antitubercular drug delivery, Colloids Surf. B: Biointerfaces 145 (2016) 679-687.

[44] A. Schoubben, P. Blasi, S. Giovagnoli, M. Ricci, C. Rossi, Simple and scalable method for peptide inhalable powder production, Eur. J. Pharm. Sci. 39 (2010) $53-58$.

[45] R. Sharma, P. Muttil, A.B. Yadav, S.K. Rath, V.K. Bajpai, U. Mani, A. Misra, Uptake of inhalable microparticles affects defence responses of macrophages infected with Mycobacterium tuberculosis H37Ra, J. Antimicrob. Chemother. 59 (2007) 499-506.

[46] T. Parumasivam, S.S. Leung, D.H. Quan, J.A. Triccas, W.J. Britton, H.K. Chan, Rifapentine-loaded PLGA microparticles for tuberculosis inhaled therapy: preparation and in vitro aerosol characterization, Eur. J. Pharm. Biopharm. 88 (2016) $1-11$.

[47] R. Diab, J. Brillault, A. Bardy, A.V. Gontijo, J.C. Olivier, Formulation and in vitro characterization of inhalable polyvinyl alcohol-free rifampicin-loaded PLGA microspheres prepared with sucrose palmitate as stabilizer: efficiency for ex vivo alveolar macrophage targeting, Int. J. Pharm. 436 (2012) 833-839.

[48] K. Tomoda, S. Kojima, M. Kajimoto, D. Watanabe, T. Nakajima, K. Makino, Effects of pulmonary surfactant system on rifampicin release from rifampicin-loaded PLGA microspheres, Colloids Surf. B: Biointerfaces 45 (2005) 1-6.

[49] V. Patomchaiviwat, O. Paeratakul, P. Kulvanich, Formation of inhalable rifampicin-poly(L-lactide) microparticles by supercritical anti-solvent process, AAPS PharmSciTech 9 (2008) 1119-1129.

[50] R. Kaur, T. Garg, B. Malik, U.D. Gupta, P. Gupta, G. Rath, A.K. Goyal, Development and characterization of spray-dried porous nanoaggregates for pulmonary delivery of anti-tubercular drugs, Drug Deliv. 23 (2016) 882-887.

[51] A.K. Goyal, T. Garg, G. Rath, U.D. Gupta, P. Gupta, Development and character ization of nanoembedded microparticles for pulmonary delivery of antitubercular drugs against experimental tuberculosis, Mol. Pharm. 12 (2015) 3839-3850.

[52] T. Garg, A.K. Goyal, G. Rath, R.S. Murthy, Spray-dried particles as pulmonary delivery system of anti-tubercular drugs: design, optimization, in vitro and in vivo evaluation, Pharm. Dev. Technol. (2015) 1-10.

[53] K. Ohashi, T. Kabasawa, T. Ozeki, H. Okada, One-step preparation of rifampicin/ poly(lactic-co-glycolic acid) nanoparticle-containing mannitol microspheres using a four-fluid nozzle spray drier for inhalation therapy of tuberculosis, J. Control. Release 135 (2009) 19-24.

[54] P.S. Pourshahab, K. Gilani, E. Moazeni, H. Eslahi, M.R. Fazeli, H. Jamalifar, Preparation and characterization of spray dried inhalable powders containing chitosan nanoparticles for pulmonary delivery of isoniazid, J. Microencapsul. 28 (2011) 605-613.

[55] B. Patel, N. Gupta, F. Ahsan, Particle engineering to enhance or lessen particle uptake by alveolar macrophages and to influence the therapeutic outcome, Eur. J. Pharm. Biopharm. 89 (2015) 163-174.

[56] K. Hirota, T. Hasegawa, H. Hinata, F. Ito, H. Inagawa, C. Kochi, G. Soma, K. Makino, H. Terada, Optimum conditions for efficient phagocytosis of rifampicin-loaded PLGA microspheres by alveolar macrophages, J. Control. Release 119 (2007) 69-76.

[57] J.A. Champion, S. Mitragotri, Role of target geometry in phagocytosis, Proc. Natl. Acad. Sci. U. S. A. 103 (2006) 4930-4934.

[58] J.-W. Yoo, S. Mitragotri, Polymer particles that switch shape in response to a stimulus, Proc. Natl. Acad. Sci. U. S. A. 107 (2010) 11205-11210.

[59] C.d.S. Bitencourt, L.B.d. Silva, P.A.T. Pereira, G.M. Gelfuso, L.H. Faccioli, Microspheres prepared with different co-polymers of poly(lactic-glycolic acid) (PLGA) or with chitosan cause distinct effects on macrophages, Colloids Surf. B Biointerfaces 136 (2015) 678-686.

[60] J.H. Park, H.E. Jin, D.D. Kim, S.J. Chung, W.S. Shim, C.K. Shim, Chitosan microspheres as an alveolar macrophage delivery system of ofloxacin via pulmonary inhalation, Int. J. Pharm. 441 (2013) 562-569.

[61] A. Alves, J. Cavaco, F. Guerreiro, J. Lourenco, A. Rosa Da Costa, A. Grenha, Inhalable Antitubercular therapy mediated by locust bean gum microparticles Molecules 21 (2016) 702.

[62] S. Tiwari, A.P. Chaturvedi, Y.B. Tripathi, B. Mishra, Macrophage-specific targeting of isoniazid through mannosylated gelatin microspheres, AAPS PharmSciTech 12 (2011) 900-908.

[63] N.K. Jain, V. Mishra, N.K. Mehra, Targeted drug delivery to macrophages, Expert Opin. Drug Deliv, 10 (2013) 353-367.

[64] I. Takeuchi, Y. Taniguchi, Y. Tamura, K. Ochiai, K. Makino, Effects of l-leucine on PLGA microparticles for pulmonary administration prepared using spray drying: fine particle fraction and phagocytotic ratio of alveolar macrophages, Colloids Surf. A Physicochem. Eng. Asp. 537 (2018) 411-417.

[65] I. Coowanitwong, V. Arya, P. Kulvanich, G. Hochhaus, Slow release formulations of inhaled rifampin, AAPS J. 10 (2008) 342-348.

[66] S. Giovagnoli, P. Blasi, A. Schoubben, C. Rossi, M. Ricci, Preparation of large porous biodegradable microspheres by using a simple double-emulsion method for capreomycin sulfate pulmonary delivery, Int. J. Pharm. 333 (2007) 103-111.

[67] H. Zhou, Y. Zhang, D.L. Biggs, M.C. Manning, T.W. Randolph, U. Christians,
B.M. Hybertson, K.Y. Ng, Microparticle-based lung delivery of INH decreases INH metabolism and targets alveolar macrophages, J. Control. Release 107 (2005) 288-299.

[68] S. Giovagnoli, F. Palazzo, A. Di Michele, A. Schoubben, P. Blasi, M. Ricci, The influence of feedstock and process variables on the encapsulation of drug suspensions by spray-drying in fast drying regime: the case of novel antitubercular drug-palladium complex containing polymeric microparticles, J. Pharm. Sci. 103 (2014) 1255-1268.

[69] F. Palazzo, S. Giovagnoli, A. Schoubben, P. Blasi, C. Rossi, M. Ricci, Development of a spray-drying method for the formulation of respirable microparticles containing ofloxacin-palladium complex, Int. J. Pharm. 440 (2013) 273-282.

[70] R. Parikh, S. Dalwadi, P. Aboti, L. Patel, Inhaled microparticles of antitubercular antibiotic for in vitro and in vivo alveolar macrophage targeting and activation of phagocytosis, J. Antibiot. 67 (2014) 387-394.

[71] A.J. Kundawala, V.A. Patel, H.V. Patel, D. Choudhary, Preparation of microparticles containing rifampicin as dry powder formulation, In: Vitro Studies on Aerosol Performance, Am. J. PharmTech Res. 2 (2012) 471-483.

[72] M.V. Bhavya, D.V. Gowda, Atul Srivastava, A.S. Aravind Ram, Riyaz Ali M. Osmani, Development and characterization of ethionamide loaded microparticles as dry powder inhalers for multi-drug resistant tuberculosis, Pharm. Lett. 8 (2016) 273-279.

[73] A.J. Kundawala, V.A. Patel, H.V. Patel, D. Choudhary, Treating tuberculosis with chitosan microparticles loaded with rifampicin as respirable powder for pulmonary delivery, Indian J. Novel Drug Deliv. 4 (2012) 57-65.

[74] R.V. Pai, R.R. Jain, A.S. Bannalikar, M.D. Menon, Development and evaluation of chitosan microparticles based dry powder inhalation formulations of rifampicin and rifabutin, J. Aerosol Med. Pulm. Drug Deliv. 29 (2016) 179-195.

[75] A.J. Kundawala, V.A. Patel, H.V. Patel, D. Choudhary, Influence of formulation components on aerosolization properties of isoniazid loaded chitosan microspheres, Int. J. Pharm. Sci. Res. 3 (2011) 297-302.

[76] P.M. Oliveira, B.N. Matos, P.A.T. Pereira, T. Gratieri, L.H. Faccioli, M.S.S. CunhaFilho, G.M. Gelfuso, Microparticles prepared with 50-190 kDa chitosan as promising non-toxic carriers for pulmonary delivery of isoniazid, Carbohydr. Polym. 174 (2017) 421-431.

[77] S. Rodrigues, A.D. Alves, J.S. Cavaco, J.F. Pontes, F. Guerreiro, A.M. Rosa Da Costa, F. Buttini, A. Grenha, Dual antibiotherapy of tuberculosis mediated by inhalable locust bean gum microparticles, Int. J. Pharm. 529 (2017) 433-441.

[78] H. Feng, L. Zhang, C. Zhu, Genipin crosslinked ethyl cellulose-chitosan complex microspheres for anti-tuberculosis delivery, Colloids Surf. B: Biointerfaces 103 (2013) 530-537.

[79] S. Tiwari, A.P. Chaturvedi, Y.B. Tripathi, B. Mishra, Microspheres based on mannosylated lysine-co-sodium alginate for macrophage-specific delivery of isoniazid, Carbohydr. Polym. 87 (2012) 1575-1582.

[80] C. Lawlor, M.P. O'Sullivan, B. Rice, P. Dillon, P.J. Gallagher, S. O'Leary, S. Shoyele, J. Keane, S.A. Cryan, Therapeutic aerosol bioengineering of targeted, inhalable microparticle formulations to treat Mycobacterium tuberculosis (MTb), J. Mater. Sci. Mater. Med. 23 (2012) 89-98.

[81] K. Hirota, T. Hasegawa, T. Nakajima, H. Inagawa, C. Kohchi, G. Soma, K. Makino, H. Terada, Delivery of rifampicin-PLGA microspheres into alveolar macrophages is promising for treatment of tuberculosis, J. Control. Release 142 (2010) 339-346.

[82] T. Hasegawa, K. Hirota, K. Tomoda, F. Ito, H. Inagawa, C. Kochi, G.-I. Soma, K. Makino, H. Terada, Phagocytic activity of alveolar macrophages toward polystyrene latex microspheres and PLGA microspheres loaded with anti-tuberculosis agent, Colloids Surf. B: Biointerfaces 60 (2007) 221-228.

[83] T. Hasegawa, K. Jijima, K. Hirota, T. Nakajima, K. Makino, H. Terada, Exact determination of phagocytic activity of alveolar macrophages toward polymer microspheres by elimination of those attached to the macrophage membrane, Colloids Surf. B: Biointerfaces 63 (2008) 209-216.

[84] K. Hirota, T. Kawamoto, T. Nakajima, K. Makino, H. Terada, Distribution and deposition of respirable PLGA microspheres in lung alveoli, Colloids Surf. B: Biointerfaces 105 (2013) 92-97.

[85] A. Yoshida, M. Matumoto, H. Hshizume, Y Oba, T. Tomishige, H. Inagawa, C. Kohchi, M. Hino, F. Ito, K. Tomoda, T. Nakajima, K. Makino, H. Terada, H. Hori, G. Soma, Selective delivery of rifampicin incorporated into poly(DL-lactic-co-glycolic) acid microspheres after phagocytotic uptake by alveolar macrophages, and the killing effect against intracellular Mycobacterium bovis Calmette-Guerin, Microbes Infect. 8 (2006) 2484-2491.

[86] S. Suarez, P. O'Hara, M. Kazantseva, C.E. Newcomer, R. Hopfer, D.N. McMurray, A.J. Hickey, Respirable PLGA microspheres containing rifampicin for the treatment of tuberculosis: screening in an infectious disease model, Pharm. Res. 18 (2001) 1315-1319.

[87] L. Garcia-Contreras, V. Sethuraman, M. Kazantseva, V. Godfrey, A.J. Hickey, Evaluation of dosing regimen of respirable rifampicin biodegradable microspheres in the treatment of tuberculosis in the guinea pig, J. Antimicrob. Chemother. 58 (2006) 980-986.

[88] J. Kaur, P. Muttil, R.K. Verma, K. Kumar, A.B. Yadav, R. Sharma, A. Misra, A handheld apparatus for "nose-only" exposure of mice to inhalable microparticles as a dry powder inhalation targeting lung and airway macrophages, Eur. J. Pharm. Sci. 34 (2008) 56-65.

[89] R.K. Verma, J. Kaur, K. Kumar, A.B. Yadav, A. Misra, Intracellular time course, pharmacokinetics, and biodistribution of isoniazid and rifabutin following pulmonary delivery of inhalable microparticles to mice, Antimicrob. Agents Chemother. 52 (2008) 3195-3201.

[90] R.K. Verma, J.K. Mukker, R.S.P. Singh, K. Kumar, P.R.P. Verma, A. Misra, Partial biodistribution and pharmacokinetics of isoniazid and rifabutin following pulmonary delivery of inhalable microparticles to rhesus macaques, Mol. Pharm. 9 
(2012) 1011-1016

[91] A.B. Yadav, P. Muttil, A.K. Singh, R.K. Verma, M. Mohan, A.K. Agrawal, A.S. Verma, S.K. Sinha, A. Misra, Microparticles induce variable levels of activation in macrophages infected with Mycobacterium tuberculosis, Tuberculosis 90 (2010) 188-196.

[92] A.B. Yadav, R. Sharma, P. Muttil, A.K. Singh, R.K. Verma, M. Mohan, S.K. Patel, A. Misra, Inhalable microparticles containing isoniazid and rifabutin target mac rophages and 'stimulate the phagocyte' to achieve high efficacy, Indian J. Exp. Biol. 47 (2009) 469-474.

[93] R. Sharma, A.B. Yadav, P. Muttil, H. Kajal, A. Misra, Inhalable microparticles modify cytokine secretion by lung macrophages of infected mice, Tuberculosis 91 (2011) 107-110.

[94] Y. Phalguna, B.S. Venkateshwarlu, K.G. Ganesh, S. Debnath, HPMC Microspheres of Zidovudine for Sustained Release, nt. J. Pharm. Pharm. Sci. 2 (2010) 41-43.

[95] H. Yamamoto, Y. Kuno, S. Sugimoto, H. Takeuchi, Y. Kawashima, Surface-modified PLGA nanosphere with chitosan improved pulmonary delivery of calcitonin by mucoadhesion and opening of the intercellular tight junctions, J. Control. Release 102 (2005) 373-381.

[96] M.A. Elgadir, M.S. Uddin, S. Ferdosh, A. Adam, A.J.K. Chowdhury, M.Z.I. Sarker, Impact of chitosan composites and chitosan nanoparticle composites on various drug delivery systems: a review, J. Food Drug Anal. 23 (2015) 619-629.

[97] I.D. Bianco, J. Balsinde, D.M. Beltramo, L.F. Castagna, C.A. Landa, E.A. Dennis, Chitosan-induced phospholipase A2 activation and arachidonic acid mobilization in P388D1 macrophages, FEBS Lett. 466 (2000) 292-294.

[98] R. Kaur, T. Garg, U. Das Gupta, P. Gupta, G. Rath, A.K. Goyal, Preparation and characterization of spray-dried inhalable powders containing nanoaggregates for pulmonary delivery of anti-tubercular drugs, Artif. Cells Nanomed. Biotechnol. 44 (2016) 182-187.

[99] T. Rawal, R. Parmar, R.K. Tyagi, S. Butani, Rifampicin loaded chitosan nanoparticle dry powder presents an improved therapeutic approach for alveolar tuberculosis, Colloids Surf. B: Biointerfaces 154 (2017) 321-330.

[100] M.I. Ahmad, T. Nakpheng, T. Srichana, The safety of ethambutol dihydrochloride dry powder formulations containing chitosan for the possibility of treating lung tuberculosis, Inhal. Toxicol. 26 (2014) 908-917.

[101] M.I. Ahmad, S. Ungphaiboon, T. Srichana, The development of dimple-shaped chitosan carrier for ethambutol dihydrochloride dry powder inhaler, Drug Dev. Ind. Pharm. 41 (2015) 791-800.

[102] R.K. Verma, A.K. Singh, M. Mohan, A.K. Agrawal, P.R. Verma, A. Gupta, A. Misra, Inhalable microparticles containing nitric oxide donors: saying NO to intracellular Mycobacterium tuberculosis, Mol. Pharm. 9 (2012) 3183-3189.
[103] R.K. Verma, A.K. Agrawal, A.K. Singh, M. Mohan, A. Gupta, P. Gupta, U.D. Gupta, A. Misra, Inhalable microparticles of nitric oxide donors induce phagosome maturation and kill Mycobacterium tuberculosis, Tuberculosis 93 (2013) 412-417.

[104] A. Gupta, G. Pant, K. Mitra, J. Madan, M.K. Chourasia, A. Misra, Inhalable particles containing rapamycin for induction of autophagy in macrophages infected with Mycobacterium tuberculosis, Mol. Pharm. 11 (2014) 1201-1207.

[105] A. Gupta, D. Sharma, J. Meena, S. Pandya, M. Sachan, S. Kumar, K. Singh, K. Mitra, S. Sharma, A.K. Panda, P. Gupta, U.D. Gupta, A. Misra, Preparation and preclinical evaluation of inhalable particles containing rapamycin and anti-tuberculosis agents for induction of autophagy, Pharm. Res. 33 (2016) 1899-1912.

[106] A. Gupta, J. Meena, D. Sharma, P. Gupta, U.D. Gupta, S. Kumar, S. Sharma, A.K. Panda, A. Misra, Inhalable particles for "pincer therapeutics" targeting nitazoxanide as bactericidal and host-directed agent to macrophages in a mouse model of tuberculosis, Mol. Pharm. 13 (2016) 3247-3255.

[107] W. Wu, C.Z. Jiang, V.A.L. Roy, Designed synthesis and surface engineering strategies of magnetic iron oxide nanoparticles for biomedical applications, Nanoscale 8 (2016) 19421-19474.

[108] A.I. Gonçalves, M.S. Miranda, M.T. Rodrigues, R.L. Reis, M.E. Gomes, Magnetic responsive cell-based strategies for diagnostics and therapeutics, Biomed. Mater. 13 (2018) 054001.

[109] P. Dames, B. Gleich, A. Flemmer, K. Hajek, N. Seidl, F. Wiekhorst, D. Eberbeck, I. Bittmann, C. Bergemann, T. Weyh, L. Trahms, J. Rosenecker, C. Rudolph, Targeted delivery of magnetic aerosol droplets to the lung, Nat. Nanotechnol. 2 (2007) 495-499.

[110] G. Hasenpusch, J. Geiger, K. Wagner, O. Mykhaylyk, F. Wiekhorst, L. Trahms, A. Heidsieck, B. Gleich, C. Bergemann, M.K. Aneja, C. Rudolph, Magnetized aerosols comprising superparamagnetic iron oxide nanoparticles improve targeted drug and gene delivery to the lung, Pharm. Res. 29 (2012) 1308-1318.

[111] F. Tewes, C. Ehrhardt, A.M. Healy, Superparamagnetic iron oxide nanoparticles (SPIONs)-loaded Trojan microparticles for targeted aerosol delivery to the lung, Eur. J. Pharm. Biopharm. 86 (2014) 98-104.

[112] A.K. Hauser, R.J. Wydra, N.A. Stocke, K.W. Anderson, J.Z. Hilt, Magnetic nanoparticles and nanocomposites for remote controlled therapies, J. Control. Release 219 (2015) 76-94.

[113] M.S. Miranda, M.T. Rodrigues, R.M.A. Domingues, R.R. Costa, E. Paz, C. Rodríguez-Abreu, P. Freitas, B.G. Almeida, M.A. Carvalho, C. Gonçalves, C.M. Ferreira, E. Torrado, R.L. Reis, J. Pedrosa, M.E. Gomes, Development of inhalable superparamagnetic iron oxide nanoparticles (SPIONs) in microparticulate system for antituberculosis drug delivery, Adv. Healthc. Mater. 7 (2018) 1800124. 